
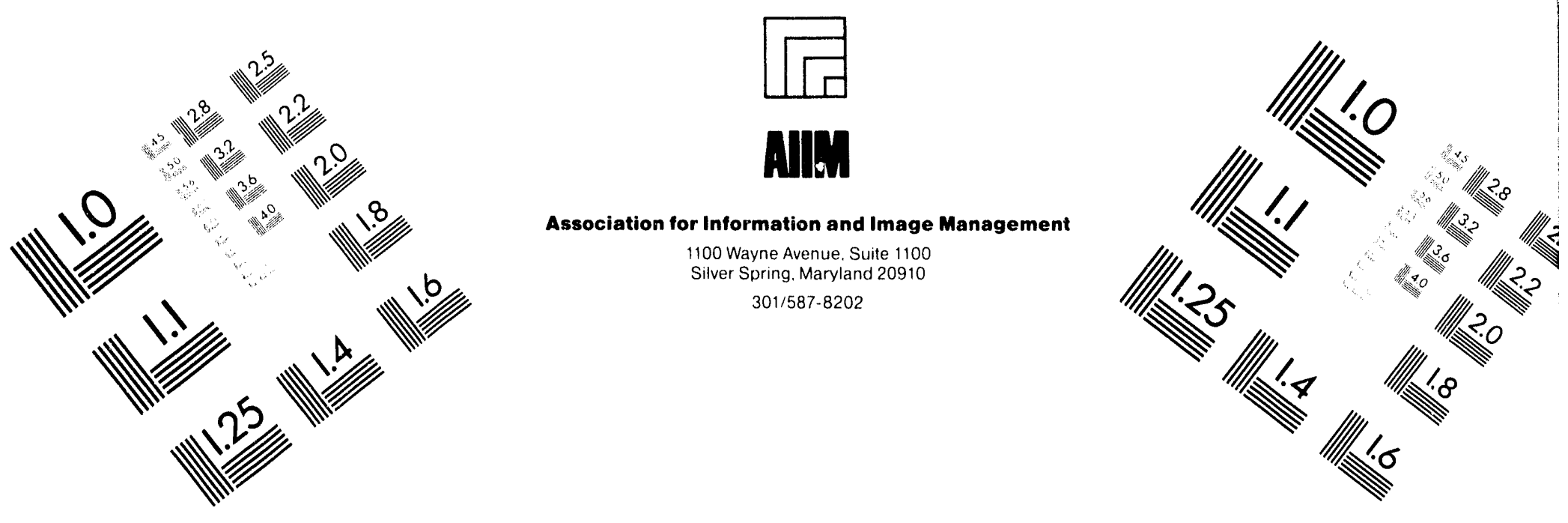

\title{
Centimeter
}

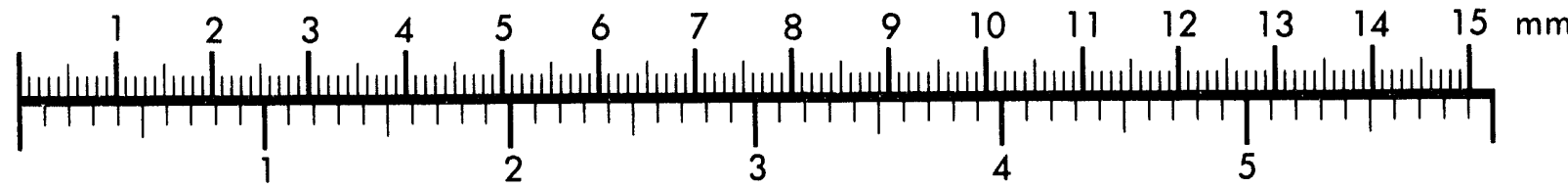

Inches
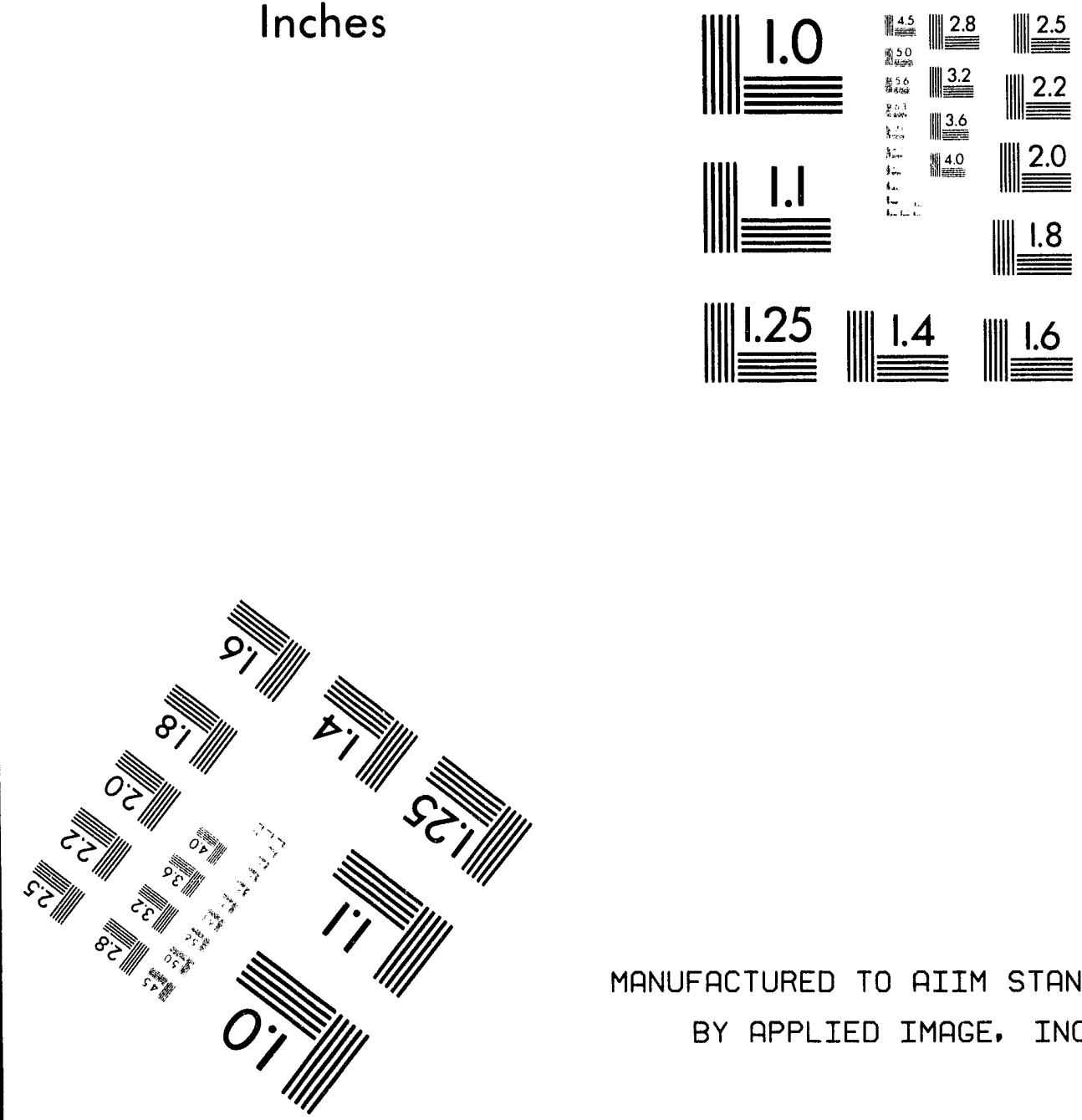

MANUFACTURED TO AIIM STANDARDS

BY APPLIED IMAGE, INC.

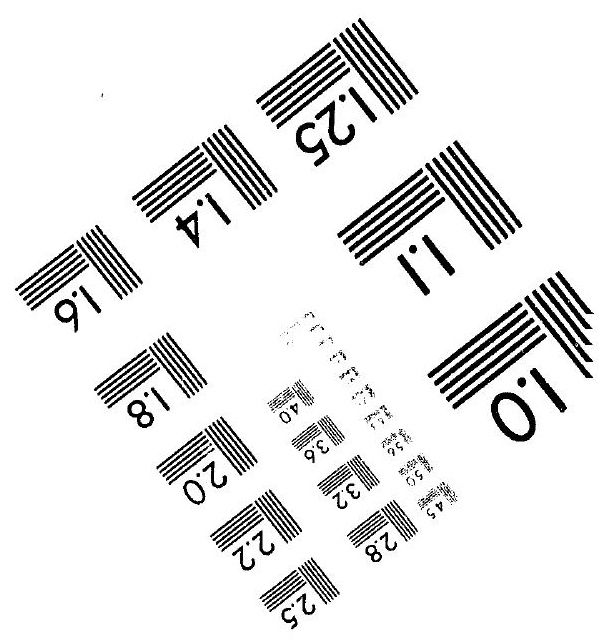



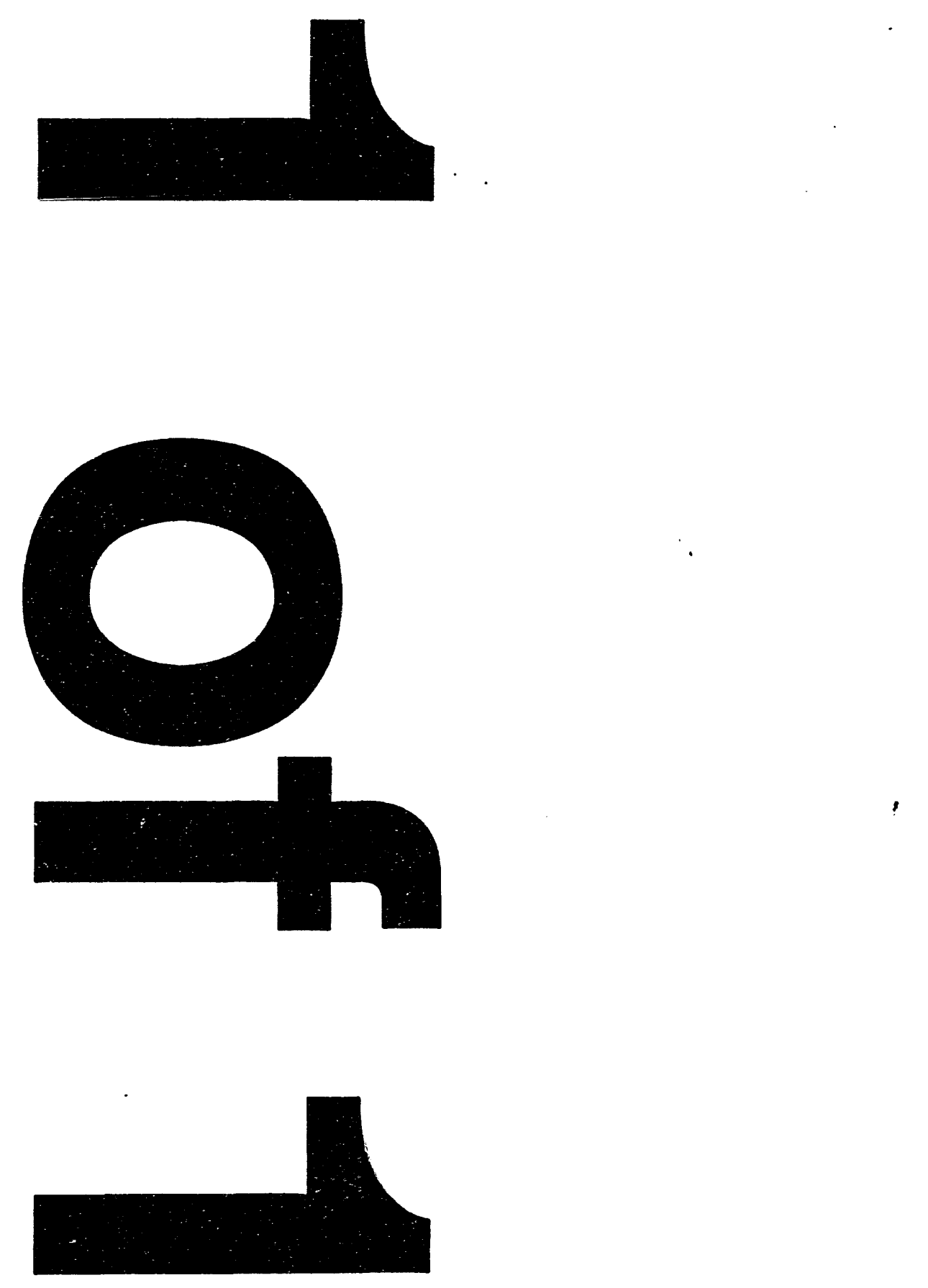
BNL-52420

UC-406

\section{DYNAMIC RESPONSE OF RIGID TANKS WITH INHOMOGENEOUS LIQUIDS}

P. Shivakumar, A. Veletsos and K. Bandyopadhyay

April 1994

Prepared for

OFFICE OF ENVIRONMENTAL RESTORATION AND WASTE MANAGEMENT DEPARTMENT OF ENERGY, WASHINGTON, D.C. 


\begin{abstract}
A study of the free vibrational characteristics and of the response to horizontal ground shaking of a rigid cylindrical tank containing an inviscid liquid with a continuous vertical variation in density is presented. The response quantities examined include the vertical sloshing motions of the liquid at its free-surface, and the impulsive and convective components of the hydrodynamic wall pressures and associated tank forces. The equations of motion for the system are formulated for an arbitrary variation in liquid density but the solutions presented are for a density that increases exponentially from top to bottom. Comprehensive numerical data are included which elucidate the underlying response mechanisms and the effects and relative importance of the various parameters involved. The solution for the continuous density variation considered herein is also compared with a previously reported solution in which the liquid was modeled as a multi-layered, discrete system.
\end{abstract}




\section{TABLE OF CONTENTS}

Section

Page

ABSTRACT $\ldots \ldots \ldots \ldots \ldots \ldots \ldots \ldots \ldots \ldots \ldots$ ii

TABLE OF CONTENTS $\ldots \ldots \ldots \ldots \ldots \ldots$

LIST OF FIGURES $\ldots \ldots \ldots \ldots \ldots \ldots \ldots \ldots \ldots$ vii

LIST OF TABLES $\ldots \ldots \ldots \ldots \ldots \ldots \ldots \ldots \ldots \ldots$ viii

EXECUTIVE SUMMARY $\ldots \ldots \ldots \ldots \ldots \ldots \ldots \ldots$ ix

ACKNOWLEDGMENT $\ldots \ldots \ldots \ldots \ldots \ldots \ldots \ldots \ldots \ldots$ xi

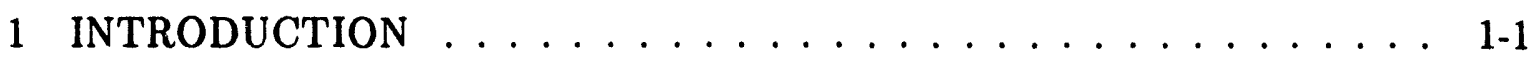

2 SYSTEM CONSIDERED $\ldots \ldots \ldots \ldots \ldots \ldots \ldots \ldots \ldots \ldots$

3 GOVERNING EQUATIONS $\ldots \ldots \ldots \ldots \ldots \ldots \ldots$ 3-1

3.1 Background Information . . . . . . . . . . . 3-1

3.2 Equation of Motion in Terms of a Single Unknown . . . . . . 3-2

3.3 Relationship with Layered Systems _ . . . . . . . . . 3-4

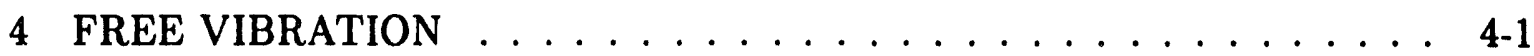

4.1 Orthogonality of Modes $\ldots \ldots \ldots \ldots \ldots \ldots$ 4-3

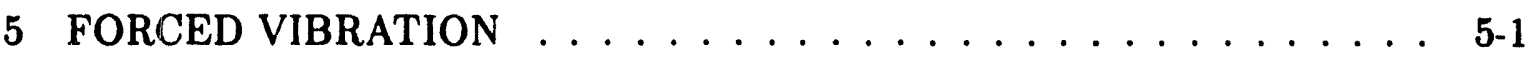

5.1 Vertical Sloshing Displacements . . . . . . . . . . . 5-2

5.2 Hydrodynamic Pressures . . . . . . . . . . . . . . . 5-2

5.2.1 Simplification for impulsive pressures $\ldots \ldots \ldots$. . . . . .

5.2 .2 Wall pressures . . . . . . . . . . . . . 5 5-4

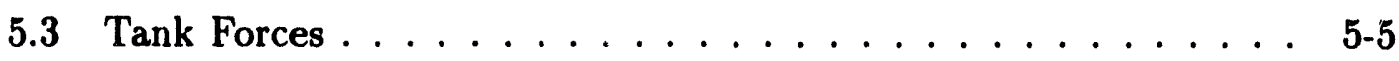

5.3 .1 Base shear . . . . . . . . . . . . 5 5-5

5.3.2 Moment above base $\ldots \ldots \ldots \ldots \ldots$. . . . . . . 5-5

5.3 .3 Foundation moment $\ldots \ldots \ldots \ldots \ldots$. . . . . . . $5-6$ 
6 NUMERICAL SOLUTIONS . . . . . . . . . . . . . . 6-1

6.1 Sloshing Frequencies and Modes . . . . . . . . . 6-1

6.2 Sloshing Displacement Coefficients . . . . . . . . . . 6-2

6.3 Hydrodynamic Pressures . . . . . . . . . . . . . . 6. 6-3

6.4 Hydrodynamic Tank Forces . . . . . . . . . . . . . . 6-4

7 CONCLUSIONS $\ldots \ldots \ldots \ldots \ldots \ldots \ldots \ldots \ldots \ldots \ldots \ldots \ldots \ldots$

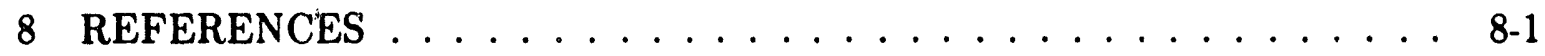

9 NOTATION ....................... . . . . 


\section{LIST OF FIGURES}

Figure

Page

2.1 System considered . . . . . . . . . . . . . . . . 2-2

2.2 Vertical variations of liquid density for different values of $\rho_{1} / \rho_{o}$ and $\beta \quad 2-3$

6.1 Frequency coefficients $C_{1 n}$ for the fundamental horizontal mode of vibration of systems with different values of $H / R$ and $\rho_{1} / \rho_{o} \ldots \ldots$ 6-6

6.2 Vertical modes of vibration associated with fundamental horizontal mode of systems with $H / R=1 \ldots \ldots$ 6-7

6.3 Surface displacement coefficients for fundamental horizontal mode of vibration of systems with different values of $H / R$ and $\rho_{1} / \rho_{o} \ldots \ldots$ 6-8

6.4 Effect of liquid density ratio $\rho_{1} / \rho_{o}$ on coefficients for impulsive and convective components of wall pressure for systems with $H / R=1.0$.

6.5 Coefficients $\bar{c}_{o}(\eta)$ and $\bar{c}_{11}(\eta)$ in expressions for impulsive and fundamental convective components of wall pressure for systems with $H / R$

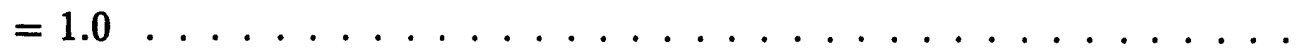

6.6 Normalized values of impulsive and fundamental convective masses for systems with different $H / R$ and $\rho_{1} / \rho_{0} \ldots \ldots \ldots$

6.7 Normalized values of coefficients for impulsive and fundamental convective components of base moment for systems with different $H / R$ and $\rho_{1} / \rho_{0} \ldots \ldots \ldots \ldots \ldots \ldots \ldots \ldots$

6.8 Normalized values of coefficients for impulsive and fundamental convective components of foundation moment for systems with different $H / R$ and $\rho_{1} / \rho_{0} \ldots \ldots \ldots \ldots \ldots \ldots$ 


\section{LIST OF TABLES}

Table

Page

6.1 Values of $\gamma_{1 n n}$ for systems with different $H / R$ and $\rho_{1} / \rho_{0} \ldots \ldots$ 6-14

6.2 Surface displacement coefficients for systems with different $H / R$ and

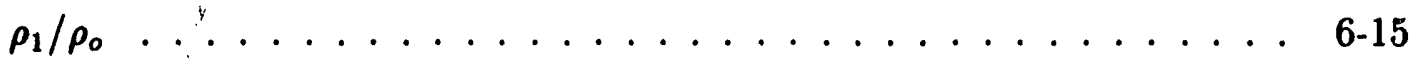

6.3 Normalized values of effective masses in expression for base shear of systems with different $H / R$ and $\rho_{1} / \rho_{0} \ldots \ldots$. . . . . . . . . .

6.4 Normalized values of coefficients in expression for overturning moment at a section immediately above tank base of systems with different $H / R$

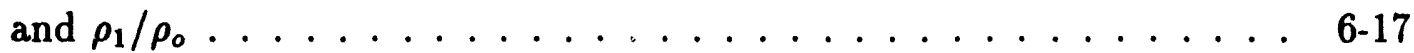

6.5 Normalized values of coefficients in expression for foundation moment of systems with different $H / R$ and $\rho_{1} / \rho_{0} \ldots \ldots \ldots$ 6-18 


\section{EXECUTIVE SUMMARY}

The study reported herein is motivated by the need for improved understanding of the response to earthquakes of tanks in nuclear facilities that store high-level radioactive wastes. It deals with the response to horizontal ground shaking of rigid vertical circular cylindrical tanks containing incompressible and inviscid liquids the densities of which increase continuously from top to bottom. The study complements those of layered liquids reported previously in Brookhaven National Laboratory Reports 52378 and 52417. Both the impulsive and convective effects are examined.

The equations of motion for the system are first formulated for liquids with an arbitrary vertical variation in density, but the solutions presented are for liquids the densities of which increase exponentially from top to bottom. In addition to the free vibrational characteristics of the contained liquid, the response quantities examined include its sloshing motion at the free-surface, and the impulsive and convective components of the hydrodynamic wall pressures and associated tank forces. Comprehensive numerical solutions are presented which elucidate the effects and relative importance of the numerous parameters involved and the relationship of these solutions to those obtained for an equivalent homogeneous system. Some attention is also given to the interrelationship of the solutions for the continuous density variation considered herein and those for its discretized, multi-layered representation reported previously.

The principal conclusions of the study are as follows :

1. Unlike a homogeneous liquid, which for a given horizontal natural mode of vibration has a single vertical mode, the inhomogeneous liquid examined has an infinite number of such modes, each associated with a distinct frequency. The latter frequencies are smaller than the corresponding frequency of the homogeneous liquid.

2. For any horizontal mode of vibration, the $n$th vertical mode of the inhomogeneous liquid has $n-1$ zero crossings, and its frequency decreases with increasing values of $n$.

3. For a specified horizontal mode of vibration, any two vertical modes satisfy a simple orthogonality relationship. 
4. When normalized with respect to the pressures computed on the assumption that the entire liquid acts as a rigid mass, the coefficients in the expression for the impulsive and all convective components of the hydrodynamic wall pressures add up to unity. The same is also true of the corresponding coefficients for base shear and base moments in the tank.

5. The impulsive component of response may be obtained either by evaluating all the convective components and subtracting their sum from the response computed on the assumption that the entire liquid acts as a rigid mass, or, independently, without the prior evaluation of the convective effects.

6. When normalized with respect to the result computed on the assumption that the entire liquid in the tank acts as a rigid mass, the total hydrodynamic wall force or base shear for an inhomogeneous liquid with values of $\rho_{1} / \rho_{0}$ in the range between 1 and 0.25 may be considered to be equal to that for a homogeneous liquid. The same is also true of the moment induced above the tank base. These two effects may, therefore, be evaluated from well-established procedures for homogeneous liquids. This approximation, however, may not be adequate for the foundation moment, particularly for broad tanks with high gradients in liquid density.

7. The finite-difference representations of the equations for the response of the continuous system examined here are the same as the response expressions for the discrete, layered system presented in the above-referenced BNL reports. 


\section{ACKNOWLEDGMENT}

This study was carried out at Rice University in cooperation with Brookhaven National Laboratory (BNL). The authors are grateful to the Department of Energy Program Directors John Tseng, James Antizzo, Howard Eckert and Dinesh Gupta for supporting the study, and to Morris Reich of BNL for his encouragement and support. 


\section{SECTION 1}

\section{INTRODUCTION}

A sequel to two recent contributions [1,2], the study reported herein is motivated by the need for improved understanding of the response to earthquakes of tanks in nuclear facilities that store high-level radioactive wastes. In some cases, the contents of these tanks cannot adequately be modeled as homogeneous liquids, and it is necessary to consider more complex representations.

In a series of recent studies $[1,2,3]$, the tank contents have been modeled as discrete, layered systems of two or more homogeneous liquid layers of different thicknesses and densities. The more general of the reported analyses $[1,2]$ permit consideration of an arbitrary number of layers, and may also be used to approximate the response of systems with continuous variations in density. For specific forms of density variations, however, it is possible to obtain simpler analytical solutions, and it is the purpose of this report to present such solutions for cylindrical tanks for which the density of the contained liquid increases exponentially from top to bottom.

The governing equations of motion are first formulated for systems with an arbitrary vertical variation in liquid density, but the solutions presented are limited to the exponential variation. Both the free-vibrational characteristics of the system and its response to a horizontal ground shaking are studied. The response quantities examined include the vertical sloshing motions of the liquid at its free-surface, and the impulsive and convective components of the hydrodynamic wall pressures and associated tank forces. The impulsive effects reflect the action of the part of the liquid that may be considered to move in synchronism with the tank wall as a rigidly attached mass, whereas the convective effects represent the action of the part of the liquid undergoing sloshing motions. Comprehensive numerical solutions are presented which elucidate the effects and relative importance of the numerous parameters involved and the relationship of these solutions to those obtained for an equivalent homogeneous system. The interrelationship of the solutions for the continuous density variation considered herein and those for its discretized, multi-layered representation is also identified. 


\section{SECTION 2}

\section{SYSTEM CONSIDERED}

The system investigated is shown in Figure 2.1. It is a rigid, vertical, circular cylindrical tank of radius $R$ that is filled to a height $H$ with an inhomogeneous liquid the mass density of which increases continuously from top to bottom. The liquid is presumed to be incompressible, irrotational and inviscid, and only linear actions are examined. The tank is considered to be anchored to a rigid, horizontally moving base. Points within the tank-liquid system are specified by the cylindrical coordinates, $r, \theta$ and $z$, as shown in the figure. The heightwise variation of the liquid density, $\rho(z)$, is defined by

$$
\rho(z)=\rho_{0} e^{-\beta(z / H)}
$$

where $\rho_{o}$ represents the density value at the tank base, and $\beta$ is a dimensionless, positive decay factor. Figure 2.2 shows the variations of $\rho(z)$ for different values of $\rho_{1} / \rho_{o}$, where $\rho_{1}$ represents the top value of the liquid density. The corresponding values of $\beta$ are shown in parentheses.

The exciting motion is considered to be uniform over the tank base and to be directed along the $\theta=0$ coordinate axis. The acceleration of the base motion at any time $t$ is denoted by $\ddot{x}_{g}(t)$, and the corresponding velocity and displacement are denoted by $\dot{x}_{g}(t)$ and $x_{g}(t)$, respectively. 


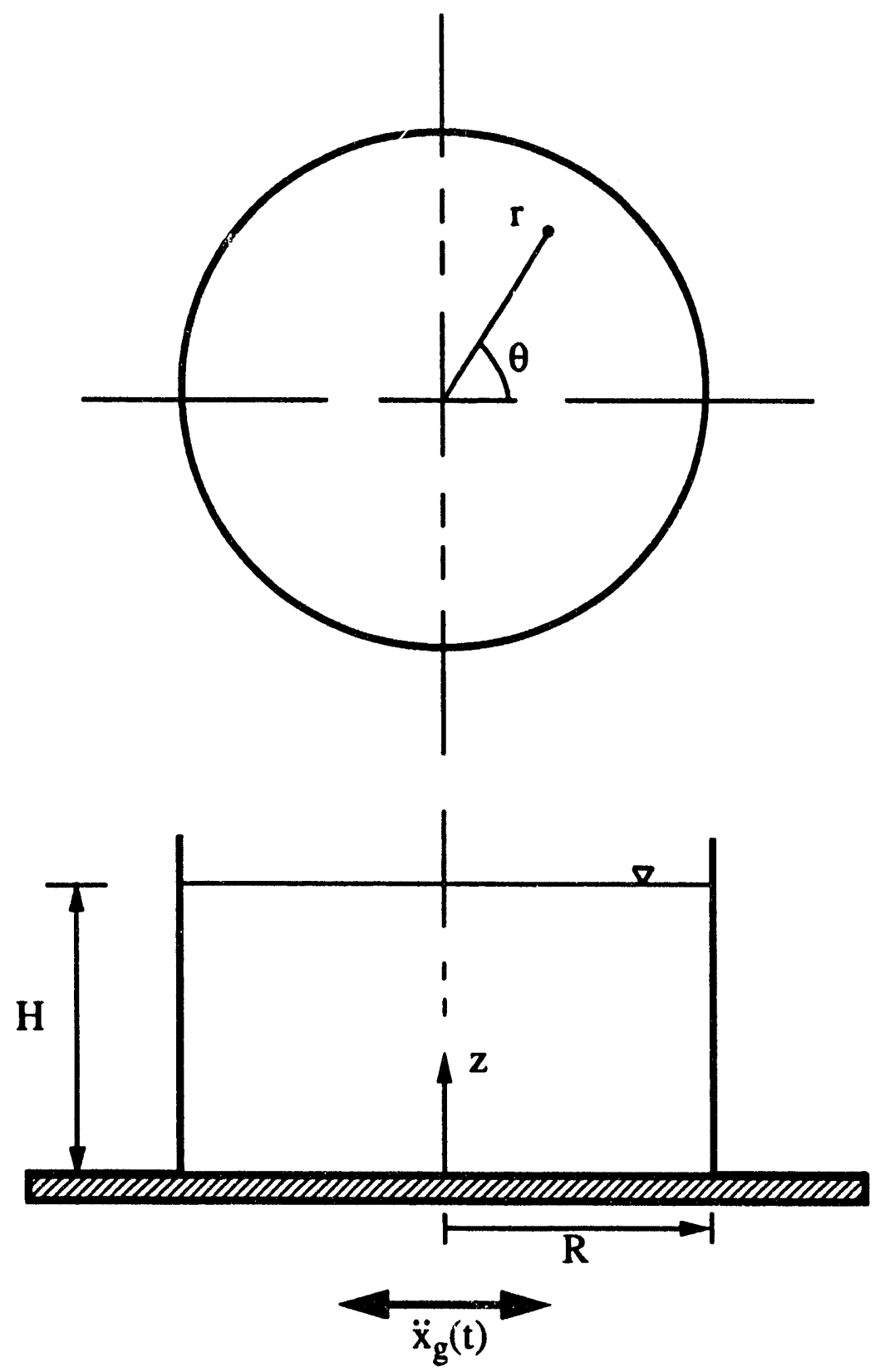

Figure 2.1 System Considered 


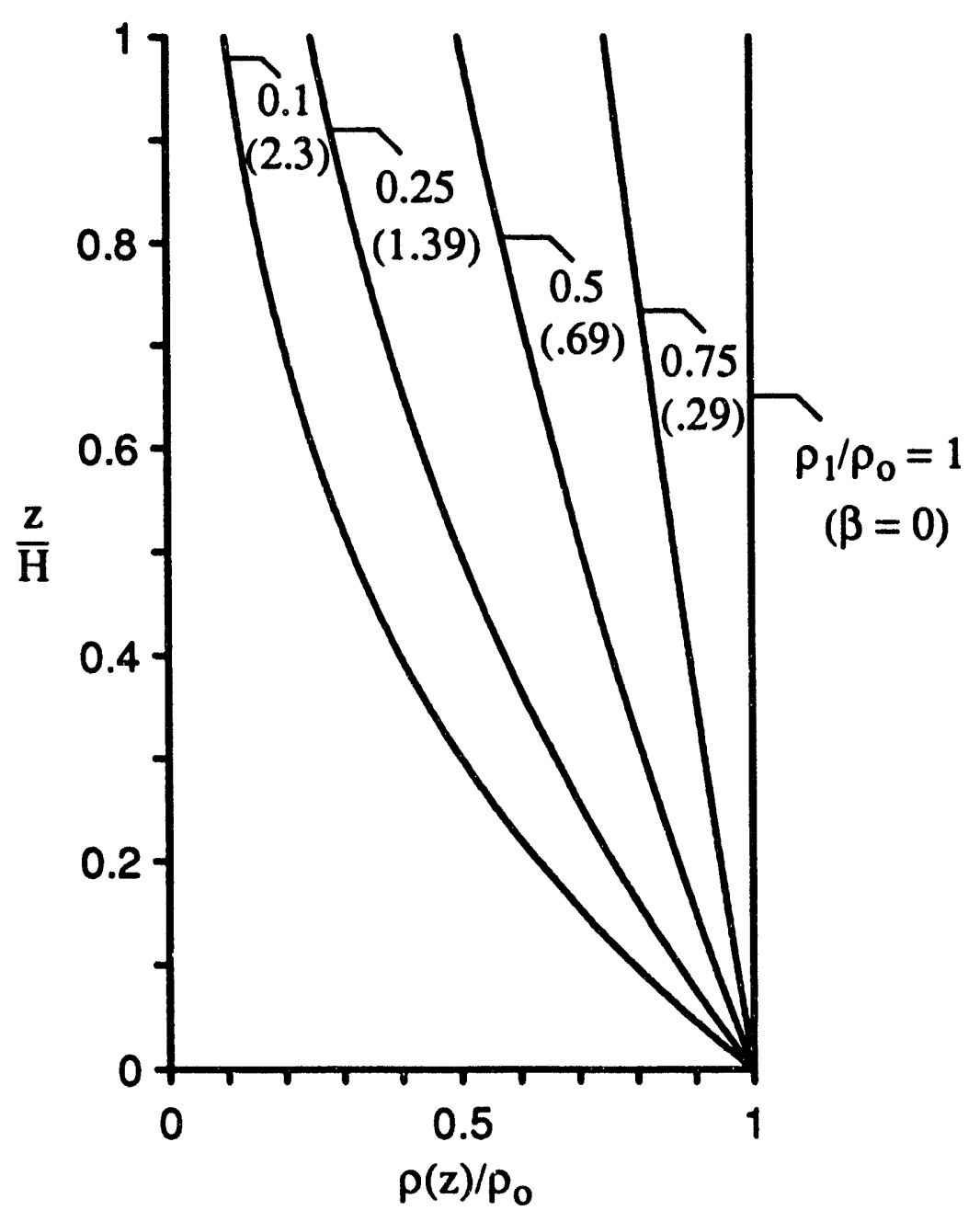

Figure 2.2 Vertical variations of liquid density for different values of $\rho_{1} / \rho_{0}$ and $\beta$ 


\section{SECTION 3}

\section{GOVERNING EQUATIONS}

\subsection{Background Information}

The response of the liquid is governed by the system of differential equations,

$$
\frac{\partial^{2} \phi}{\partial r^{2}}+\frac{1}{r} \frac{\partial \phi}{\partial r}+\frac{1}{r^{2}} \frac{\partial^{2} \phi}{\partial \theta^{2}}-\frac{\partial^{2} d}{\partial z \partial t}=0
$$

and

$$
\frac{\partial}{\partial z}\left(\rho \frac{\partial \phi}{\partial t}\right)+\rho \frac{\partial^{2} d}{\partial t^{2}}-\frac{\partial \rho}{\partial z} g d=0
$$

in which $d=d(r, z, \theta, t)$ is the vertical sloshing displacement of the liquid at an arbitrary point and time, and $\phi=\phi(r, z, \theta, t)$ is a velocity potential function which is related to the hydrodynamic pressure, $p=p(r, z, \theta, t)$, by

$$
p=\rho \frac{\partial \phi}{\partial t}
$$

and to the radial and tangential components of $i$ quid velocity, $v_{r}$ and $v_{\theta}$, by

$$
v_{r}=-\frac{\partial \phi}{\partial r} \quad v_{\theta}=-\frac{1}{r} \frac{\partial \phi}{\partial \theta}
$$

Equations (2) and (3) are deduced from more general expressions presented by Yih [4] by expressing the latter in cylindrical coordinates and specializing them to the incompressible liquid considered herein. For a homogeneous liquid with $\rho=$ constant,

$$
\frac{\partial d}{\partial t}=-\frac{\partial \phi}{\partial z}
$$

and equation (2) reduces to the well-known Laplace's equation $\nabla^{2} \phi=0$.

The solutions of equations (2) and (3) must satisfy the continuity of radial velocities at the tank-wall, defined by

$$
\left(\frac{\partial \phi}{\partial r}\right)_{r=R}=-\dot{x}_{g}(t) \cos \theta
$$

the condition of no vertical motion at the tank-base, defined by

$$
(d)_{z=0}=0
$$

and the linearized pressure condition at the free liquid surface, defined by

$$
\left(\frac{\partial \phi}{\partial t}-g d\right)_{z=H}=0
$$

where $g$ is the acceleration of gravity. 


\subsection{Equation of Motion in Terms of a Single Unknown}

Following the approach used in the analysis of the layered system examined in References 1 and 2, the potential function $\phi$ is expressed in the form

$$
\phi(r, \theta, z, t)=-\dot{x}_{g}(t) r \cos \theta+\psi(r, \theta, z, t)
$$

where the first term on the right side provides for the rigid body motion of the tank, and the potential function $\psi$ provides for the relative motion of the liquid and tank. On substituting equation (10) into equations (2) and (3), one obtains

$$
\frac{\partial^{2} \psi}{\partial r^{2}}+\frac{1}{r} \frac{\partial \psi}{\partial r}+\frac{1}{r^{2}} \frac{\partial^{2} \psi}{\partial \theta^{2}}-\frac{\partial^{2} d}{\partial z \partial t}=0
$$

and

$$
\frac{\partial}{\partial z}\left(\rho \frac{\partial \psi}{\partial t}\right)+\rho \frac{\partial^{2} d}{\partial t^{2}}-\frac{\partial \rho}{\partial z} g d=\frac{\partial \rho}{\partial z} r \ddot{x}_{g}(t) \cos \theta
$$

The solutions of equations (11) and (12) may be obtained by the method of separation of variables in the form

$$
\begin{aligned}
& d(r, z, \theta, t)=D(z, t) X(r) \cos \theta \\
& \psi(r, z, \theta, t)=\Psi(z, t) X(r) \cos \theta
\end{aligned}
$$

That the function $X(r)$ in these two expressions must be the same follows from equation (12). On substituting equations (13) and (14) into equation (11), separating the resulting functions of $r$ from those of $z$ and $t$, and equating each set to $-(\lambda / R)^{2}$, where $\lambda$ is a dimensionless constant, one obtains Bessel's differential equation for $X(r)$ and the following relation between $\Psi$ and $D$ :

$$
\Psi(z, t)=-\frac{R^{2}}{\lambda^{2}} \frac{\partial^{2} D}{\partial z \partial t}
$$

Next, on using the antisymmetry condition at $r=0$ and the continuity condition for radial velocities at $r=R$, the solution for $X(r)$ can be shown to be given by any one of an infinity of Bessel functions of the first kind and first order. The $m$ th of these functions may be expressed as

$$
X_{m}(r)=B_{m} J_{1}\left(\lambda_{m} \frac{r}{R}\right)
$$

where $B_{m}$ is a constant that remains to be determined, and $\lambda_{m}$ is the $m$ th root of the first derivative of $J_{1}(\lambda)$, the first three values of which are

$$
\lambda_{1}=1.841 \quad \lambda_{2}=5.331 \quad \lambda_{3}=8.536
$$


Equation (16) effectively defines the radial variation of the displacement $d$ when the liquid is oscillating in its $m$ th horizontal mode of vibration. The displacement $d$ at an arbitrary point and time is then determined from equation (13) as a linear combination of its modal components to be

$$
d(\xi, \eta, \theta, t)=\sum_{m=1}^{\infty} D_{m}(\eta, t) \frac{J_{1}\left(\lambda_{m} \xi\right)}{J_{1}\left(\lambda_{m}\right)} \cos \theta
$$

where the constant $B_{m}$ in equation (16) has been absorbed into the function $D_{m}$; $\xi=r / R$ and $\eta=z / H$ are dimensionless radial and vertical position coordinates; and $D_{m}(\eta, t)$ represents the instantaneous value of the vertical displacement of a liquid particle at the junction of the tank-wall and the $\theta=0$ plane when the liquid is vibrating in its $m$ th horizontal natural mode. The corresponding expression for $\psi$ is determined from equations (14), (15) and (16) to be

$$
\psi(\xi, \eta, \theta, t)=-\sum_{m=1}^{\infty} \frac{R^{2}}{\lambda_{m}^{2} H} \frac{\partial \dot{D}_{m}}{\partial \eta} \frac{J_{1}\left(\lambda_{m} \xi\right)}{J_{1}\left(\lambda_{m}\right)} \cos \theta
$$

where a dot superscript denotes differentiation with respect to time.

On substituting equations (18) and (19) into equation (12) and making use of the orthogonality of the Bessel functions, the equation of motion for the system can be expressed solely in terms of the function $D_{m}(\eta, t)$, as

$\rho \frac{\partial^{2} \ddot{D}_{m}}{\partial \eta^{2}}+\frac{\partial \rho}{\partial \eta} \frac{\partial \ddot{D}_{m}}{\partial \eta}-\rho\left(\frac{\lambda_{m} H}{R}\right)^{2} \ddot{D}_{m}+\frac{g \lambda_{m}}{R}\left(\frac{\lambda_{m} H}{R}\right) \frac{\partial \rho}{\partial \eta} D_{m}=-\epsilon_{m} \lambda_{m}\left(\frac{\lambda_{m} H}{R}\right) \frac{\partial \rho}{\partial \eta} \ddot{x}_{g}(t)$

where

$$
\epsilon_{m}=\frac{2}{\lambda_{m}^{2}-1}
$$

Furthermore, on making use of equations (10), (18) and (19), the boundary conditions defined by equations (8) and (9) reduce to

$$
\begin{gathered}
\left(D_{m}\right)_{z=0}=0 \\
\left(\frac{R^{2}}{\lambda_{m}^{2} H} \frac{\partial \ddot{D}_{m}}{\partial \eta}+g D_{m}\right)_{z=H}=-\epsilon_{m} R \ddot{x}_{g}(t)
\end{gathered}
$$

After determining the functions $D_{m}=D_{m}(\eta, t)$ for different values of $m$, the displacement $d$, the potential functions $\psi$ and $\phi$, and the hydrodynamic pressure $p$ may be computed from equations (18), (19), (10) and (4), respectively. 
For the system with the exponential variation in liquid density considered herein, equation $(20)$ reduces tc

$$
\frac{\partial^{2} \ddot{D}_{m}}{\partial \eta^{2}}-\beta \frac{\partial \ddot{D}_{m}}{\partial \eta}-\left(\frac{\lambda_{m} H}{R}\right)^{2} \ddot{D}_{m}-\frac{g \lambda_{m}}{R}\left(\frac{\lambda_{m} H}{R}\right) \beta D_{m}=\epsilon_{m} \lambda_{m}\left(\frac{\lambda_{m} H}{R}\right) \beta \ddot{x}_{g}(t)
$$

\subsection{Relationship with Layered Systems}

It is instructive to compare equation (19) for the continuous system with the corresponding expression for the discrete, layered system examined in Reference 1 . The potential function $\psi_{j}$ for the $j$ th layer of the latter system is given by

$$
\psi_{j}=-\sum_{m=1}^{\infty} \frac{R}{\lambda_{m}}\left[\frac{\dot{D}_{m, j} \cosh \lambda_{m} \eta_{j}-\dot{D}_{m, j-1} \cosh \lambda_{m}\left(\alpha_{j}-\eta_{j}\right)}{\sinh \lambda_{m} \alpha_{j}}\right] \frac{J_{1}\left(\lambda_{m} \xi\right)}{J_{1}\left(\lambda_{m}\right)} \cos \theta
$$

where $\alpha_{j}=H_{j} / R$ and $\eta_{j}=z_{j} / R$. Note that unlike the distance coordinate $\eta$ employed in the analysis of the continuous system, which is normalized with respect to the liquid depth, $H$, the coordinate $\eta_{j}$ is normalized with respect to the tank radius, $R$. Note further that as the layer-height, $H_{j}=\Delta z$, is decreased, $\alpha_{j}=\Delta z / R$ tends to zero, the cosh functions in equation (25) tend to unity, the sinh function tends to $\lambda_{m} \Delta z / R=\lambda_{m}(H / R) \Delta \eta$, and equation (25) becomes the finite-difference counterpart of equation (19). It can similarly be shown that the equations of motion for the layered system (equation 28 of Reference 1) are merely the finite-difference counterparts of equation (20). It follows that, contrary to the view expressed in Reference 5, the representation of the continuous system as a multi-layered system is indeed a valid approximation. For a discussion of the accuracy of this representation, the reader is referred to References 2 and 6. 


\section{SECTION 4}

\section{FREE VIBRATION}

On setting the right-hand side of equation (24) equal to zero and letting $D_{m}(\eta, t)=$ $\hat{D}_{m}(\eta) e^{i \omega_{m} t}$, where $i=\sqrt{-1}$ and $\omega_{m}$ is the circular frequency of the $m$ th horizontal mode of vibration, one obtains

$$
\frac{\partial^{2} \hat{D}_{m}}{\partial \eta^{2}}-\beta \frac{\partial \hat{D}_{m}}{\partial \eta}+\left[\frac{\beta \lambda_{m}}{C_{m}^{2}} \frac{H}{R}-\left(\frac{\lambda_{m} H}{R}\right)^{2}\right] \hat{D}_{m}=0
$$

in which $C_{m}$ is a dimensionless factor related to $\omega_{m}$ by

$$
\omega_{m}=C_{m} \sqrt{\frac{g \lambda_{m}}{R}}
$$

Similarly, the boundary conditions defined by equations (22) and (23) can be expressed as

$$
\begin{gathered}
\left(\hat{D}_{m}\right)_{\eta=0}=0 \\
\left(\frac{\partial \hat{D}_{m}}{\partial \eta}-\frac{\lambda_{m}}{C_{m}^{2}} \frac{H}{R} \hat{D}_{m}\right)_{\eta=1}=0
\end{gathered}
$$

With appropriate reinterpretations of the symbols involved, equations (26), (28) and (29) can be shown to be the same as those presented by Lamb [7] for the flow of inhomogeneous liquids in rectangular channels.

The nature of the solution of equation (26) depends on whether the roots of the associated characteristic equation are real-valued or complex, and this depends, in turn, on the value of $C_{m}$. For

$$
C_{m}>\sqrt{\frac{\beta\left(\lambda_{m} H / R\right)}{\beta^{2} / 4+\left(\lambda_{m} H / R\right)^{2}}}
$$

the roots are real, and on satisfying the boundary condition defined by equation (28), the solution can be written as

$$
\hat{D}_{m}(\eta)=E_{m} e^{\beta \eta / 2} \sinh \left(\gamma_{m} \eta\right)
$$

where

$$
\gamma_{m}=\sqrt{\frac{\beta^{2}}{4}+\left(\frac{\lambda_{m} H}{R}\right)^{2}-\frac{\beta \lambda_{m} H / R}{C_{m}^{2}}}
$$


and $E_{m}$ is an arbitrary constant. Note that $\gamma_{m}$ is a function of the still unknown frequency coefficient $C_{m}$. On making use of the second boundary condition defined by equation (29), it is found that $\gamma_{m}$ and $C_{m}$ are also interrelated by

$$
C_{m}=\sqrt{\frac{\lambda_{m} H / R}{\beta / 2+\gamma_{m} / \tanh \gamma_{m}}}
$$

and on eliminating $C_{m}$ from equations (32) and (33), one obtains the transcendental equation

$$
\gamma_{m}^{2}+\frac{\beta \gamma_{m}}{\tanh \gamma_{m}}+\frac{\beta^{2}}{4}-\left(\lambda_{m} \frac{H}{R}\right)^{2}=0
$$

It can be shown that equation (34) has a single positive root, $\gamma_{m 1}$, and that this root exists only if

$$
\frac{\beta^{2}}{4}+\beta-\left(\lambda_{m} \frac{H}{R}\right)^{2}<0
$$

With the value of $\gamma_{m 1}$ established, the displacement configuration for the $m$ th horizontal and first vertical natural mode of vibration, $\hat{D}_{m 1}(\eta)$, is determined from equation (31) by replacing the subscript $m$ by $m 1$, and the associated circular natural frequency, $\omega_{m 1}$, and frequency coefficient, $C_{m 1}$, are determined similarly from equations (27) and (33), respectively. It should be recalled that equations (31) and (33) are valid only as long as equation (35) is satisfied.

For values of $C_{m}$ that are smaller than the right-hand member of equation (30), the roots of the characteristic equation are complex, and the counterpart of equation (34) becomes

$$
\gamma_{m}^{2}-\frac{\beta \gamma_{m}}{\tan \gamma_{m}}-\frac{\beta^{2}}{4}+\left(\lambda_{m} \frac{H}{R}\right)^{2}=0
$$

The latter equation has an infinity of roots, $\gamma_{m n}$, where $n$ is an integer ranging from 2 to $\infty$ when equation (35) is satisfied and from 1 to $\infty$ when equation (35) is not satisfied. It follows that, for each horizontal mode of vibration, there is an infinity of vertical modes, each associated with a distinct frequency. Subject to the indicated qualification on $n$, the $m$ th horizontal and $n$th vertical mode of vibration, $\hat{D}_{m n}(\eta)$, is given by

$$
\hat{D}_{m n}(\eta)=E_{m n} e^{\beta \eta / 2} \sin \left(\gamma_{m n} \eta\right)
$$

where $E_{m n}$ is an arbitrary constant, and the associated frequency, $\omega_{m n}$, may be expressed as

$$
\omega_{m n}=C_{m n} \sqrt{\frac{g \lambda_{m}}{R}}
$$


in which the dimensionless coefficient, $C_{m n}$, is determined from

$$
C_{m n}=\sqrt{\frac{\lambda_{m}(H / R)}{\beta / 2+\gamma_{m n} / \tan \gamma_{m n}}}
$$

For a homogeneous liquid for which $\beta=0$, equation (34) yields

$$
\gamma_{m}=\lambda_{m} \frac{H}{R}
$$

which when substituted into equation (33), yields the well-established expression for the frequency coefficient (see, for example, Reference 8)

$$
C_{m}=\sqrt{\tanh \left(\frac{\lambda_{m} H}{R}\right)}
$$

The associated mode of vibration is determined from equation (31) to be

$$
\hat{D}_{m}(\eta)=E_{m} \sinh \left(\lambda_{m} \frac{H}{R} \eta\right)
$$

The corresponding solutions from equations (36), (39) and (37) are trivial and are not considered.

\subsection{Orthogonality of Modes}

The modal displacements defined by equations (31) and (37) satisfy the orthogonality relation

$$
\int_{0}^{1}[\beta+\delta(\eta-1)] \rho(\eta) \hat{D}_{m r}(\eta) \hat{D}_{m s}(\eta) d \eta=0 \quad \text { for } \mathrm{r} \neq \mathrm{s}
$$

in which the term involving the delta function, $\delta(\eta-1)$, accounts for the discontinuity in the liquid density value at the free-surface. The derivation of this equation follows well-established steps. Specifically, equation $(26)$ for $\hat{D}_{m r}(\eta)$ is multiplied through by $\rho(\eta) \hat{D}_{m e}(\eta)$ and integrated from 0 to 1 ; the term involving the second derivative of $\hat{D}_{m r}(\eta)$ in the resulting expression is integrated by parts; and use is made of the boundary condition defined by equation (29) to obtain

$$
\begin{gathered}
{\left[\frac{\beta}{C_{m r}^{2}}-\frac{\lambda_{m} H}{R}\right] \int_{0}^{1} \rho(\eta) \hat{D}_{m r}(\eta) \hat{D}_{m s}(\eta) d \eta=} \\
\frac{1}{C_{m s}^{2}} \rho_{1} \hat{D}_{m r}(1) \hat{D}_{m s}(1)+\int_{0}^{1} \frac{\rho(\eta)}{\lambda_{m} H / R} \frac{\partial \hat{D}_{m r}}{\partial \eta} \frac{\partial \hat{D}_{m s}}{\partial \eta} d \eta
\end{gathered}
$$

These steps are repeated by starting with the $\hat{D}_{m o}(\eta)$ mode and multiplying through by $\rho(\eta) \hat{D}_{m r}(\eta)$. The resulting expression is then subtracted from equation (44) to obtain equation (43). 
Equation (43) may also be deduced from the corresponding expression for the $\mathrm{N}$ layered, discrete system examined in Reference 1. The latter expression is

$$
\left\{\hat{D}_{m r}\right\}^{T}[\mathcal{B}]\left\{\hat{D}_{m s}\right\}=0
$$

where $\left\{\hat{D}_{m r}\right\}$ and $\left\{\hat{D}_{m \bullet}\right\}$ are vectors of size $N$ that define the amplitudes of the interfacial displacements; and $[\mathcal{B}]$ is a matrix of size $N \times N$ expressing the values of the density discontinuities at the interfaces. To obtain equation (43), one must : (1) replace the elements of the first $(N-1)$ rows of $[\mathcal{B}]$ by $(-\partial \rho / \partial \eta) d \eta,(2)$ replace the elements of the $N$ th row by the density discontinuity at the top, $\delta(\eta-1) \rho(\eta) d \eta$, and (3) express the inner products of the modal displacement vectors as an integral from 0 to 1 of the modal displacement functions. 


\section{SECTION 5}

\section{FORCED VIBRATION}

With the natural frequencies and modes of vibration of the system established, its response to an arbitrary lateral excitation may be determined by modal superposition. In this approach, the modal displacements, $D_{m}(\eta, t)$, are expressed in the form

$$
D_{m}(\eta, t)=\sum_{n=1}^{\infty} \hat{D}_{m n}(\eta) q_{m n}(t)
$$

where $q_{m n}(t)$ is a generalized time-dependent coordinate corresponding to the $m$ th horizontal and $n$th vertical mode of vibration. Substituting equation (46) into equation (24), multiplying through by $[\beta+\delta(\eta-1)] \rho(\eta) \hat{D}_{m n}(\eta)$, and making use of the orthogonality of the natural modes defined by equation (43), one finds that $q_{m n}$ is governed by the differential equation

$$
\ddot{q}_{m n}(t)+\omega_{m n}^{2} q_{m n}(t)=-\epsilon_{m} \omega_{m n}^{2} \Gamma_{m n} R \frac{\ddot{x}_{g}(t)}{g}
$$

in which $\Gamma_{m n}$ is a dimensionless factor given by

$$
\Gamma_{m n}=\frac{\int_{0}^{1}[\beta+\delta(\eta-1)] \rho(\eta) \hat{D}_{m n}(\eta) d \eta}{\int_{0}^{1}[\beta+\delta(\eta-1)] \rho(\eta) \hat{D}_{m n}^{2}(\eta) d \eta}
$$

The solution of equation (47) is given by

$$
q_{m n}(t)=-\epsilon_{m} \Gamma_{m n} R \frac{A_{m n}(t)}{g}
$$

where $A_{m n}(t)$ represents the instantaneous pseudoacceleration of an undamped singledegree-of-freedom oscillator with a circular natural frequency $\omega_{m n}$ subjected to the prescribed ground acceleration, and is given by

$$
A_{m n}(t)=\omega_{m n} \int_{0}^{t} \ddot{x}_{g}(\tau) \sin \left[\omega_{m n}(t-\tau)\right] d \tau
$$

The maximum value of $A_{m n}(t)$ is the quantity displayed on a pseudoacceleration response spectrum. Substitution of equation (49) into equation (46) leads to

$$
D_{m}(\eta, t)=-R \sum_{n=1}^{\infty} \delta_{m n}(\eta) \frac{A_{m n}(t)}{g}
$$


where $\delta_{m n}(\eta)$, a dimensionless function corresponding to the $m$ th horizontal and $n$th vertical mode of vibration, is given by

$$
\delta_{m n}(\eta)=\epsilon_{m} \Gamma_{m n} \hat{D}_{m n}(\eta)
$$

The latter function is the counterpart of the vector of displacement coefficients $\left\{d_{m n}\right\}$ in the analysis of the layered system presented in Reference 1.

\subsection{Vertical Sloshing Displacements}

On substituting equation (51) into equation (18), the vertical displacement of the liquid at any point and time, $d(\xi, \eta, \theta, t)$, is found to be

$$
d(\xi, \eta, \theta, t)=-R \sum_{m=1}^{\infty} \sum_{n=1}^{\infty} \delta_{m n}(\eta) \frac{J_{1}\left(\lambda_{m} \xi\right)}{J_{1}\left(\lambda_{m}\right)} \frac{A_{m n}(t)}{g} \cos \theta
$$

As is true of the coefficients in the corresponding expressions for layered systems presented in References 1 and 2 , the coefficients $\delta_{m n}$ can be shown to satisfy the relations

$$
\sum_{n=1}^{\infty} \delta_{m n}(\eta)=\epsilon_{m}
$$

and

$$
\sum_{m=1}^{\infty} \sum_{n=1}^{\infty} \delta_{m n}(\eta)=1
$$

For a homogeneous liquid with $\beta=0$, for which there is only one vertical mode of vibration for each horizontal mode, the quantities $\hat{D}_{m n}(\eta), \Gamma_{m n}, \delta_{m n}(\eta)$ and $A_{m n}(t)$ are denoted by $\hat{D}_{m}(\eta), \Gamma_{m}, \delta_{m}(\eta)$ and $A_{m}(t)$, respectively. The free-surface value of $\delta_{m}$ is then evaluated from equations $(48)$ and $(52)$ to be $\delta_{m}(1)=\epsilon_{m}$, and equation (53) reduces, as it should, to (see, for example, Reference 8)

$$
d(\xi, 1, \theta, t)=-R \sum_{m=1}^{\infty} \epsilon_{m} \frac{J_{1}\left(\lambda_{m} \xi\right)}{J_{1}\left(\lambda_{m}\right)} \frac{A_{m}(t)}{g} \cos \theta
$$

\subsection{Hydrodynamic Pressures}

On substituting equation (19) into equation (10) and making use of equation (4), the hydrodynamic pressure may be expressed as

$$
p(\xi, \eta, \theta, t)=\left\{-\xi \ddot{x}_{g}(t) e^{-\beta \eta}-\frac{R}{H} \sum_{m=1}^{\infty} \frac{e^{-\beta \eta}}{\lambda_{m}^{2}}-\frac{\partial \ddot{D}_{m}(\eta, t)}{\partial \eta} \frac{J_{1}\left(\lambda_{m} \xi\right)}{J_{1}\left(\lambda_{m}\right)}\right\} \rho_{0} R \cos \theta
$$

Furthermore, on substituting equation (51) into this expression, making use of the relation

$$
\ddot{A}_{m n}(t)=\omega_{m n}^{2}\left[\ddot{x}_{g}(t)-A_{m n}(t)\right]
$$


which is obtained by substituting equation (49) into equation (47), and grouping terms with similar temporal variations, the hydrodynamic pressure can be expressed as the sum of two components : an impulsive component, $p^{i}$, given by

$$
p^{i}(\xi, \eta, \theta, t)=-\left[e^{-\beta \eta} \xi-\sum_{m=1}^{\infty} \sum_{n=1}^{\infty} c_{m n}(\eta) \frac{J_{1}\left(\lambda_{m} \xi\right)}{J_{1}\left(\lambda_{m}\right)}\right] \rho_{o} R \cos \theta \ddot{x}_{g}(t)
$$

and a convective component, $p^{c}$, given by

$$
p^{c}(\xi, \eta, \theta, t)=-\left[\sum_{m=1}^{\infty} \sum_{n=1}^{\infty} c_{m n}(\eta) \frac{J_{1}\left(\lambda_{m} \xi\right)}{J_{1}\left(\lambda_{m}\right)} A_{m n}(t)\right] \rho_{o} R \cos \theta
$$

in which

$$
c_{m n}(\eta)=\frac{R}{H} \frac{e^{-\beta \eta}}{\lambda_{m}} C_{m n}^{2} \frac{\partial \delta_{m n}(\eta)}{\partial \eta}
$$

The impulsive component represents the effect of the portion of the liquid that may be considered to move as a rigid body in synchronism with the tank wall, while the convective component represents the effect of the liquid undergoing sloshing action. It should be observed that the pressures in equations (59) and (60) are expressed in terms of the base value of the liquid density, $\rho_{o}$, rather than the density value at the height being considered.

5.2.1 Simplification for impulsive pressures. In the form presented in equation (59), the evaluation of the impulsive component of response requires the prior evaluation of the convective components. The impulsive component can also be evaluated independently of the convective by letting

$$
e_{m}(\eta)=\sum_{n=1}^{\infty} C_{m n}^{2} \delta_{m n}(\eta)
$$

and rewriting equation (59) in the form of a single series as

$$
p^{i}(\xi, \eta, \theta, t)=-e^{-\beta \eta}\left[\xi-\frac{R}{H} \sum_{m=1}^{\infty} \frac{1}{\lambda_{m}} \frac{\partial e_{m}}{\partial \eta} \frac{J_{1}\left(\lambda_{m} \xi\right)}{J_{1}\left(\lambda_{m}\right)}\right] \rho_{o} R \cos \theta \ddot{x}_{g}(t)
$$

It can be shown that the function $e_{m}(\eta)$ may be determined without prior knowledge of the $\delta_{m n}(\eta)$ functions from the differential equation

$$
\frac{\partial^{2} e_{m}}{\partial \eta^{2}}-\beta \frac{\partial e_{m}}{\partial \eta}-\left(\frac{\lambda_{m} H}{R}\right)^{2} e_{m}=-\epsilon_{m} \beta \lambda_{m} \frac{H}{R}
$$

subject to the boundary conditions

$$
\left(e_{m}\right)_{\eta=0}=0
$$


and

$$
\left(\frac{\partial e_{m}}{\partial \eta}\right)_{\eta=1}=\epsilon_{m} \lambda_{m} \frac{H}{R}
$$

Equation (64) is obtained by substituting equation (51) into equation (24), making use of the relation defined by equation (58), grouping the terms involving $\ddot{x}_{g}(t)$, and finally making use of equations (27) and (62). Equations (65) and (66) are derived by proceeding similarly with equations (22) and (23).

The solution of equation (64) is given by

$$
e_{m}(\eta)=\frac{\epsilon_{m}}{\lambda_{m} H / R}\left[b_{1} e^{a_{m 1} \eta}+b_{2} e^{a_{m 2} \eta}+\beta\right]
$$

where

$$
\begin{gathered}
a_{m 1, m 2}=\frac{\beta}{2} \pm \sqrt{\frac{\beta^{2}}{4}+\left(\frac{\lambda_{m} H}{R}\right)^{2}} \\
b_{1}=\frac{\beta a_{m 2} e^{a_{m 2}}+\left(\lambda_{m} H / R\right)^{2}}{a_{m 1} e^{a_{m 1}}-a_{m 2} e^{a_{m 2}}}
\end{gathered}
$$

and

$$
b_{2}=-\frac{\beta a_{m 1} e^{a_{m 1}}+\left(\lambda_{m} H / R\right)^{2}}{a_{m 1} e^{a_{m 1}}-a_{m 2} e^{a_{m 2}}}
$$

5.2.2 Wall pressures. The impulsive and convective components of the hydrodynamic pressures induced against the tank-wall are determined from equations (63) and (60) by letting $\xi=1$. T.e total wall pressure at an arbitrary height may be expressed in the form

$$
p(1, \eta, \theta, t)=-\left[c_{o}(\eta) \ddot{x}_{g}(t)+\sum_{m=1}^{\infty} \sum_{n=1}^{\infty} c_{m n}(\eta) A_{m n}(t)\right] \rho_{o} R \cos \theta
$$

where the dimensionless function $c_{o}(\eta)$ for the impulsive component of the pressure is determined from equation (63) to be

$$
c_{o}(\eta)=e^{-\beta \eta}\left[1-\frac{R}{H} \sum_{m=1}^{\infty} \frac{1}{\lambda_{m}} \frac{\partial e_{m}}{\partial \eta}\right]
$$

and the corresponding functions for the convective components are determined from equation (61). From equations (61), (62) and (72), it now follows that

$$
c_{o}(\eta)+\sum_{m=1}^{\infty} \sum_{n=1}^{\infty} c_{m n}(\eta)=e^{-\beta \eta}
$$

For a homogeneous system $(\beta=0)$, equation (67) yields

$$
e_{m}(z)=\epsilon_{m} \frac{\sinh \left(\lambda_{m} z / R\right)}{\cosh \left(\lambda_{m} H / R\right)}
$$


and equation (72) reduces, as it should, to the well-established expression (see, for example, Reference 8 )

$$
c_{o}(z)=1-\sum_{m=1}^{\infty} \epsilon_{m} \frac{\cosh \left(\lambda_{m} z / R\right)}{\cosh \left(\lambda_{m} H / R\right)}
$$

in which the $m$ th term of the summation represents the coefficient $c_{m}(z)$ for the convective component of the pressure.

\subsection{Tank Forces}

5.3.1 Base shear. The base shear or total hydrodynamic force exerted on the tank wall is obtained by integrating equation (71) over the tank-height. The result may be expressed in the form

$$
Q_{b}(t)=m_{o} \ddot{x}_{g}(t)+\sum_{m=1}^{\infty} \sum_{n=1}^{\infty} m_{m n} A_{m n}(t)
$$

where $m_{o}$, the impulsive component of the liquid mass, is given by

$$
m_{o}=\left(\int_{0}^{1} c_{o}(\eta) d \eta\right) \rho_{o} \pi R^{2} H
$$

and $m_{m n}$, the convective component associated with the $m$ th horizontal and $n$th vertical sloshing mode of vibration, is given by

$$
m_{m n}=\left(\int_{0}^{1} c_{m n}(\eta) d \eta\right) \rho_{o} \pi R^{2} H
$$

From equations (77) and (78), and with the aid of equation (73), it can finally be shown that

$$
m_{o}+\sum_{m=1}^{\infty} \sum_{n=1}^{\infty} m_{m n}=\left(\int_{0}^{1} e^{-\beta \eta} d \eta\right) \rho_{o} \pi R^{2} H=m_{l}
$$

where $m_{l}$ is the total mass of the contained liquid.

The integrals in equations (77) and (78) and those in the expressions for base moments presented in the following two sections can be evaluated readily. The resulting expressions are lengthy and are not presented, but comprehensive numerical solutions for both the base shear and base moment are given in later sections.

5.3.2 Moment above base. The moment induced by the hydrodynamic wall pressure at a section of the tank immediately above its base may conveniently be expressed in the form

$$
M(t)=m_{o} h_{o} \ddot{x}_{g}(t)+\sum_{m=1}^{\infty} \sum_{n=1}^{\infty} m_{m n} h_{m n} A_{m n}(t)
$$


where the coefficient $m_{o} h_{o}$ for the impulsive component is given by

$$
m_{\circ} h_{\circ}=\left(\int_{0}^{1} c_{o}(\eta) \eta d \eta\right) \rho_{o} \pi R^{2} H^{2}
$$

and the coefficient $m_{m n} h_{m n}$ for the convective component associated with the $m$ th horizontal and $n$th vertical mode of vibration is evaluated from

$$
m_{m n} h_{m n}=\left(\int_{0}^{1} c_{m n}(\eta) \eta d \eta\right) \rho_{o} \pi R^{2} H^{2}
$$

From the latter two expressions and from equation (73), it follows that

$$
m_{o} h_{\circ}+\sum_{m=1}^{\infty} \sum_{n=1}^{\infty} m_{m n} h_{m n}=\left[\int_{0}^{1} e^{-\beta \eta} \eta d \eta\right] \rho_{o} \pi R^{2} H^{2}=m_{l} h_{l}
$$

where $m_{l} h_{l}$ represents the moment above the tank base induced by an unit horizontal acceleration when the entire liquid is presumed to act as a rigid mass.

5.3.3 Foundation moment. In addition to the moment defined by equation (80), the foundation moment, $M^{\prime}(t)$, includes the effect of the hydrodynamic pressure exerted on the tank base. The latter effect is determined by appropriate integration of the base values of the hydrodynamic pressures defined by equation (59) or (63) and equation (60). The resulting expression may be written as

$$
M^{\prime}(t)=m_{o} h_{o}^{\prime} \ddot{x}_{g}(t)+\sum_{m=1}^{\infty} \sum_{n=1}^{\infty} m_{m n} h_{m n}^{\prime} A_{m n}(t)
$$

where the coefficient $m_{o} h_{o}^{\prime}$ for the impulsive component of the moment is given by

$$
m_{o} h_{o}^{\prime}=m_{o} h_{o}+\left[\frac{1}{4}-\left.\frac{R}{H} \sum_{m=1}^{\infty} \frac{1}{\lambda_{m}^{3}} \frac{\partial e_{m}}{\partial \eta}\right|_{\eta=0}\right] \rho_{o} \pi R^{4}
$$

and the coefficient $m_{m n} h_{m n}^{\prime}$ for the convective component associated with the $m$ th horizontal and $n$th vertical mode of vibration is given by

$$
m_{m n} h_{m n}^{\prime}=m_{m n} h_{m n}+\left[\left.\frac{R}{H} \frac{C_{m n}^{2}}{\lambda_{m}^{3}} \frac{\partial d_{m n}}{\partial \eta}\right|_{\eta=0}\right] \rho_{o} \pi R^{4}
$$

From equations (85) and (86), and with the aid of equations (62) and (83), it can further be shown that

$$
m_{o} h_{o}^{\prime}+\sum_{m=1}^{\infty} \sum_{n=1}^{\infty} m_{m n} h_{m n}^{\prime}=m_{l} h_{l}+\frac{\rho_{o} \pi R^{4}}{4}=m_{l} h_{l}^{\prime}
$$

where $m_{l} h_{l}^{\prime}$ represents the foundation moment induced by a unit horizontal acceleration when the entire liquid is considered to act as a rigid mass. It should be kept in mind that the base pressure in this case increases linearly from zero at the center to $\rho_{o} R \cos \theta$ at the junction of the base and wall. 


\section{SECTION 6}

\section{NUMERICAL SOLUTIONS}

The numerical solutions presented in this section are for the free vibrational characteristics and for the response to horizontal base shaking of systems with different slenderness ratios, $H / R$, and different liquid density ratios, $\rho_{1} / \rho_{o}$.

\subsection{Sloshing Frequencies and Modes}

Mention has already been made of the fact that unlike a homogeneous liquid which for a prescribed horizontal mode of vibration has a single vertical mode, an inhomogeneous liquid has an infinite number of vertical modes, each with a distinct frequency.

Figure 6.1 shows the variation with the density ratio $\rho_{1} / \rho_{o}$ of the frequency coefficients $C_{1 n}$ for the fundamental horizontal and first three vertical modes of vibration of systems with $H / R$ values in the range between 0.5 and 2 . It is observed that the highest frequency coefficient $C_{11}$ for the fundamental horizontal and vertical mode of vibration of the inhomogeneous system is smaller than that for the corresponding homogeneous system. However, the difference is quite small, especially for larger values of $\rho_{1} / \rho_{o}$ and $H / R$. By contrast, the coefficients $C_{1 n}$ for $n \geq 2$ are significantly smaller than those for $n=1$ and quite sensitive to variations in $\rho_{1} / \rho_{o}$. These trends may be explained by examining the modal displacement amplitudes, $\hat{D}_{1 n}(\eta)$.

Figure 6.2 shows the first three vertical modes of vibration corresponding to the fundamental horizontal mode for systems with $H / R=1$ and three values of $\rho_{1} / \rho_{0}$ in the range between 0.1 and 1 . Each mode is normalized such that its maximum amplitude is unity. It is observed that the $n$th vertical mode of vibration is associated with $n-1$ zero crossings. Since the vertical motion of the liquid is zero at these sections, the natural frequency of the system for this mode must equal that of a system with the same density distribution and a depth equal to the distance from the free surface to the uppermost level of zero amplitude. For $n>1$, these effective depths are but small fractions of the total depth, $H$, and decrease with increasing $\rho_{1} / \rho_{o}$. The associated natural frequencies, which, based on equation (41) for a homogeneous liquid, are expected to be proportional to the effective liquid depth must, therefore, also be small and decrease with increasing $\rho_{1} / \rho_{o}$. For $n=1$, on the other hand, the more rapid decays in the displacement amplitudes with depth are obtained for the 
smaller values of $\rho_{1} / \rho_{0}$. A decrease in $\rho_{1} / \rho_{o}$ in this case is associated with a reduced effective depth for the system, and hence a reduced natural frequency. However, the differences are quite small, and for values of $\rho_{1} / \rho_{0}$ in the range between 0.25 and 1 , the fundamental natural frequencies of the inhomogeneous and homogeneous systems may be considered to be the same. These trends are representative of those obtained for the higher order horizontal modes of vibration (higher values of $m$ ) as well.

Table 6.1 lists the values of $\gamma_{m n}$ for the first two horizontal and first three vertical modes of vibration of systems with several combinations of $H / R$ and $\rho_{1} / \rho_{o}$. For the results marked with asterisks, the condition defined by equation (35) is not satisfied, and $\gamma_{11}$ was evaluated from equation (36) rather than from equation (34). The frequency coefficients, $C_{11}$, and modes of vibration, $\hat{D}_{11}(\eta)$, for these cases must, therefore, be evaluated from equations (39) and (37) rather than from equations (33) and (31).

\subsection{Sloshing Displacement Coefficients}

Of special interest in practice is the sloshing motion of the liquid at its free surface, as the maximum surface displacement is needed to define the freeboard that must be provided to prevent the liquid from overflowing or impacting the roof. This displacement is obtained by letting $\eta=1$ in equation (53).

The displacement coefficients $\delta_{1 n}(1)$ for the fundamental horizontal and first three vertical modes of vibration are presented in Figure 6.3. The results are plotted in a manner analogous to that employed in Figure 6.1 as a function of the density ratio $\rho_{1} / \rho_{o}$ for three values of $H / R$ in the range from 0.5 to 2 . These data along with corresponding data for additional systems and for the second horizontal mode of vibration are listed in Table 6.2. The following trends are worth noting :

1. The surface displacement coefficients are relatively insensitive to the value of $H / R$ but increase substantially with decreasing $\rho_{1} / \rho_{0}$. The latter trend is consistent with that reported in Reference 1 for layered systems, and is attributed to the fact that, the larger the variation in liquid density, the greater is the sloshing action induced.

2. The corresponding coefficients for the second horizontal mode of vibration, $m=$ 2 , are significantly smaller than those for the fundamental mode, $m=1$, and the sum of the coefficients over $n$ for each horizontal mole satisfies equation (54). Furthermore, when all the horizontal modes of vibration are considered, the algebraic sum of the coefficients, in agreement with equation (55), is unity. 
It should be realized that the relative contributions of the various modes of vibration to the surface sloshing motion depend not only on the relative values of the displacement factors $\delta_{m n}(1)$ but also on those of the corresponding pseudoaccelerations, $A_{m n}(t)$. The latter quantities depend, in turn, on the characteristics of the ground motion and the natural frequencies of the system itself.

\subsection{Hydrodynamic Pressures}

Shown in the left part of Figure 6.4 are the heightwise variations of the function $c_{o}(\eta)$ in equation (72) for the impulsive component of the hydrodynamic wall pressure. These plots are for a tank with $H / R=1$ and liquids with density ratios $\rho_{1} / \rho_{0}$ in the range between 0.1 and 1 . Also shown are the corresponding functions $c_{11}(\eta)$ and $c_{12}(\eta)$ for the fundamental horizontal and first two vertical sloshing modes. As would be expected (recall that the pressures are expressed in terms of the base value of the liquid density rather than that at the level being considered), the functions $c_{o}(\eta)$ and $c_{11}(\eta)$ decrease with decreasing $\rho_{1} / \rho_{o}$. Furthermore, consistent with the distributions of the modal displacement amplitudes displayed in Figure 6.2, the function $c_{1 n}(\eta)$ for the $n$th vertical mode of vibration exhibits $n-1$ changes in sign.

The interrelationship of the hydrodynamic response for the inhomogeneous and homogeneous systems can be better appreciated by rewriting equation (71) for the wall pressure in the form

$$
p(1, \eta, \theta, t)=-\left[\bar{c}_{o}(\eta) \ddot{x}_{g}(t)+\sum_{m=1}^{\infty} \sum_{n=1}^{\infty} \bar{c}_{m n}(\eta) A_{m n}(t)\right] \rho_{a v} R \cos \theta
$$

where $\rho_{a v}$ represents the average value of the liquid density, given by

$$
\rho_{a v}=\frac{\rho_{o}\left[1-e^{-\beta}\right]}{\beta}
$$

and the functions $\bar{c}_{o}(\eta)$ and $\bar{c}_{m n}(\eta)$ are related to the functions $c_{o}(\eta)$ and $c_{m n}(\eta)$ in equation (71) by

$$
\bar{c}_{o}(\eta)=\frac{\rho_{o}}{\rho_{a v}} c_{o}(\eta) \quad \text { and } \quad \bar{c}_{m n}(\eta)=\frac{\rho_{o}}{\rho_{a v}} c_{m n}(\eta)
$$

The variations of $\bar{c}_{0}(\eta)$ and $\bar{c}_{11}(\eta)$ are displayed in Figure 6.5 for the values of $\rho_{1} / \rho_{0}$ and $H / R$ considered previously in Figure 6.4. It is observed that these functions are still sensitive to variations in liquid density, but that for values of $\rho_{1} / \rho_{0}$ in the range between 0.25 to 1 , the areas under the individual curves are close to each other. It follows that, despite the indicated differences in the pressures themselves, the total hydrodynamic wall force or base shear for the inhomogeneous and homogeneous 
liquids can be interrelated simply. This matter is considered further in the following section.

\subsection{Hydrodynamic Tank Forces}

Figure 6.6 shows the variations with $\rho_{1} / \rho_{o}$ and $H / R$ of the impulsive and fundamental convective masses, $m_{0}$ and $m_{11}$, in the expression for the hydrodynamic base shear. These masses are normalized with respect to the total liquid mass, $m_{l}$. Normalized values of the corresponding base moment coefficients, $m_{o} h_{o}$ and $m_{11} h_{11}$, and of the foundation moment coefficients, $m_{o} h_{o}^{\prime}$ and $m_{11} h_{11}^{\prime}$, are plotted in Figures 6.7 and 6.8, respectively. It should be reemphasized that these three sets of normalized quantities express the hydrodynamic base shear and base moments as fractions of those computed on the assumption that the entire liquid acts as a rigid mass. The normalizing quantities are naturally different for tanks of different proportions and contents. The normalized values of $m_{\circ}$ and $m_{11}$ for additional systems, along with the corresponding values of $m_{12}, m_{21}$ and $m_{22}$, are presented in Table 6.3, and the normalized values of the base and foundation moment coefficients are presented in Tables 6.4 and 6.5.

Examination of the data presented in these figures and tables reveals the following trends :

1. For values of $\rho_{1} / \rho_{0}$ in the range between 1 and about 0.25 , the normalized values of $m_{o}$ and $m_{m 1}$ may, for all practical purposes, be considered to be the same. This result, which is consistent with the prediction made from the pressure profiles displayed in Figure 6.5, is generally valid over the entire range of $H / R$ examined. Incidentally, the seismic response of systems normally encountered in practice is dominated by $m_{o}$ and, to a lesser degree, by $m_{11}$ and $m_{21}$. On recalling that within the range of $\rho_{1} / \rho_{o}$ considered, the sloshing frequencies, $\omega_{m 1}$, and the spectral values of the associated pseudoaccelerations, $A_{m 1}(t)$, also are insensitive to variations in the density ratio, it is concluded that, when normalized with respect to the value computed on the assumption that the entire liquid in the tank acts as a rigid mass, the total hydrodynamic wall force or base shear for an inhomogeneous liquid is practically equal to that for a homogeneous liquid. The same is also true of the moment induced above the tank base. These two effects may, therefore, be evaluated from well-established procedures for homogeneous liquids. This approximation, however, is not adequate for the foundation moment, particularly for tanks with low values of $H / R$ and $\rho_{1} / \rho_{0}$. The latter moment is dominated by the hydrodynamic pressures exerted on the 
tank base, and, as already demonstrated, these pressures may be substantially different for inhomogeneous and homogeneous systems.

2. For values of $\rho_{1} / \rho_{0}$ less than about 0.25 , the fraction of the liquid that acts impulsively is generally smaller for the inhomogeneous system than the homogeneous system. The large density gradients in this case increase the proportions of the liquid participating in the convective or sloshing actions. This increase, however, does not necessarily increase the convective force coefficients associated with the fundamental sloshing mode of vibration. For a given horizontal mode of vibration and a prescribed response, it is the sum of the coefficients for all the vertical modes that generally increases. 


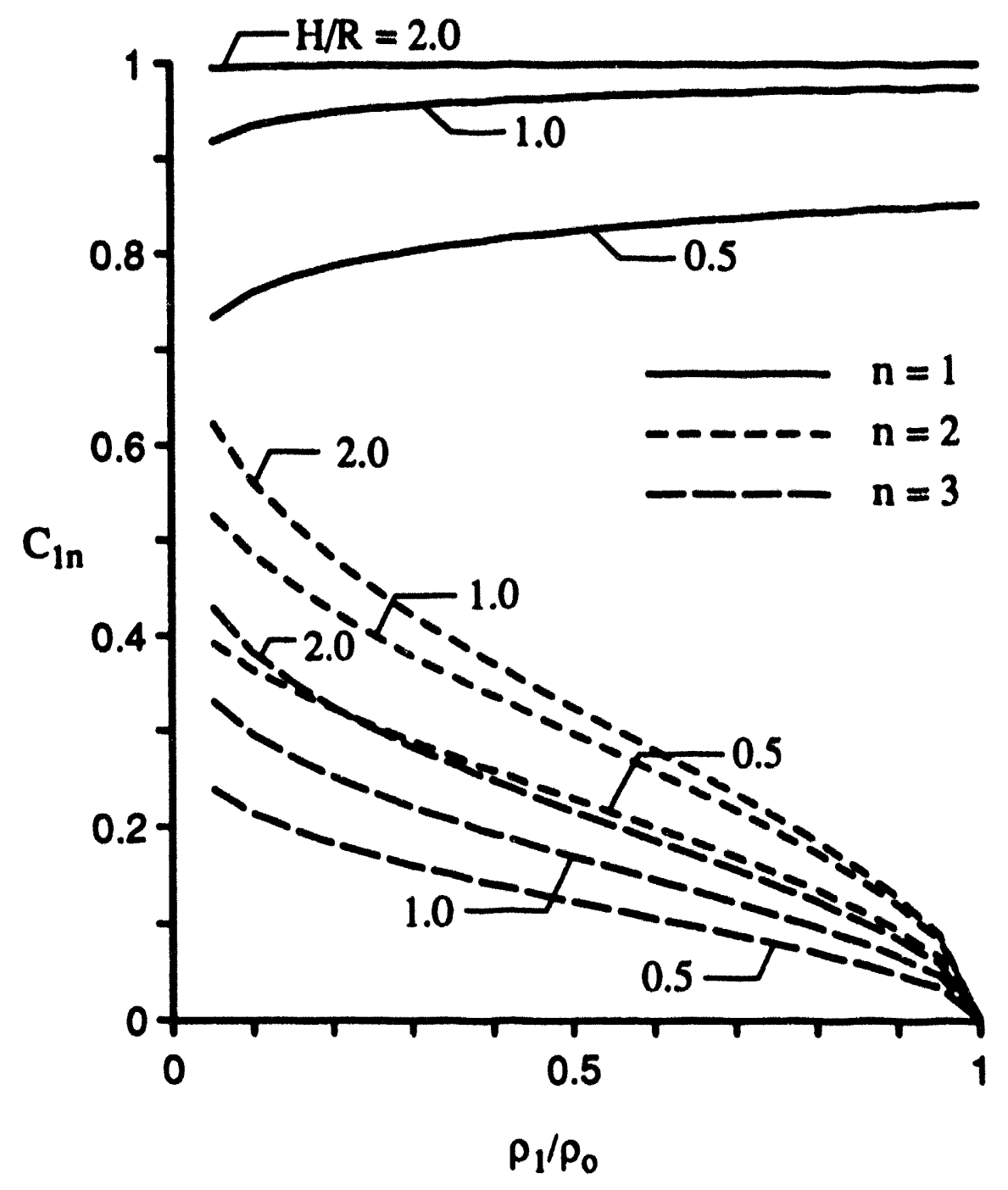

Figure 6.1 Frequency coefficients $\mathrm{C}_{1 \mathrm{n}}$ for the fundamental horizontal mode of vibration of systems with different values of $H / R$ and $\rho_{1} / \rho_{0}$ 


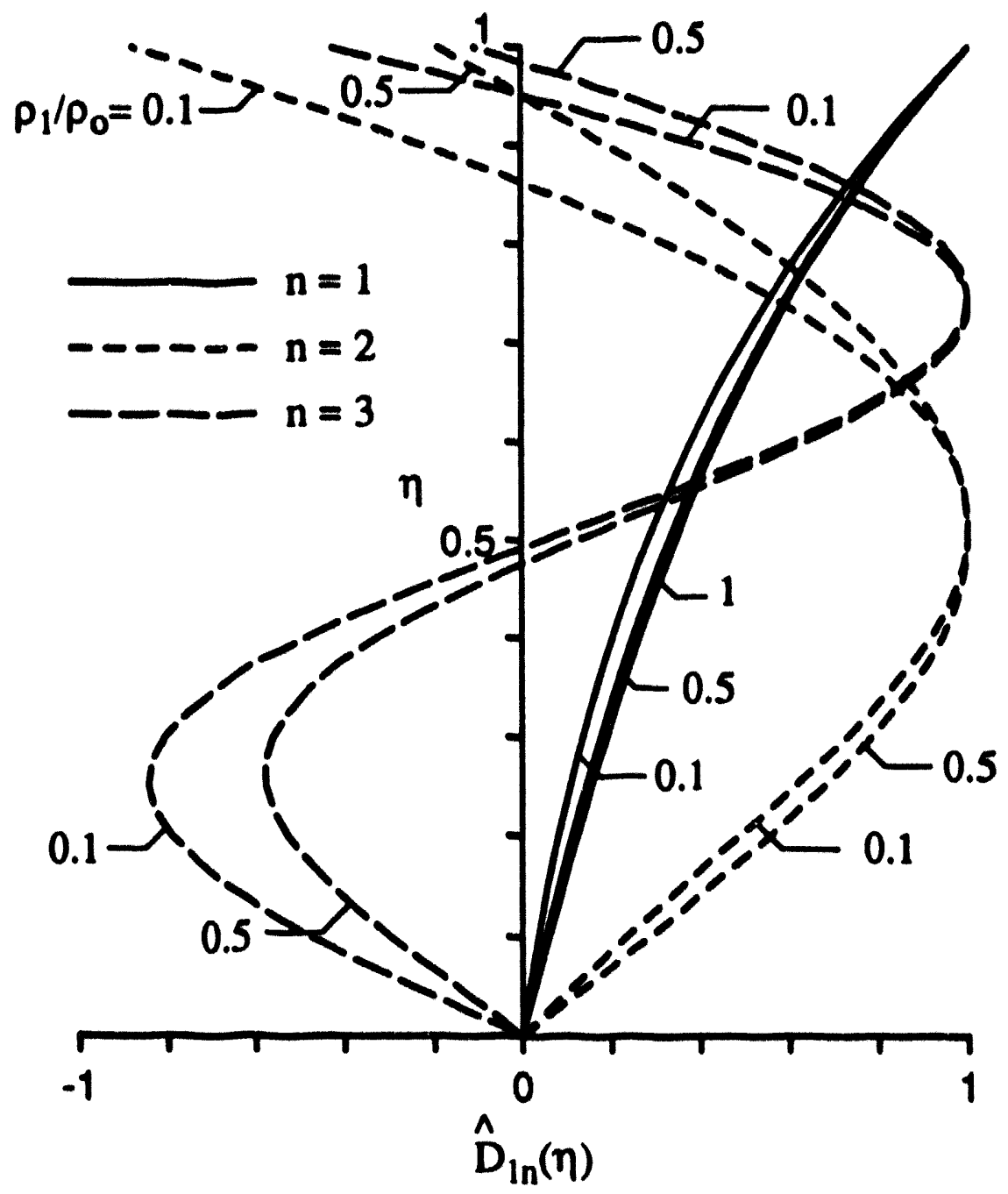

Figure 6.2 Vertical modes of vibration associated with fundamental horizontal mode of systems with $H / R=1$ 


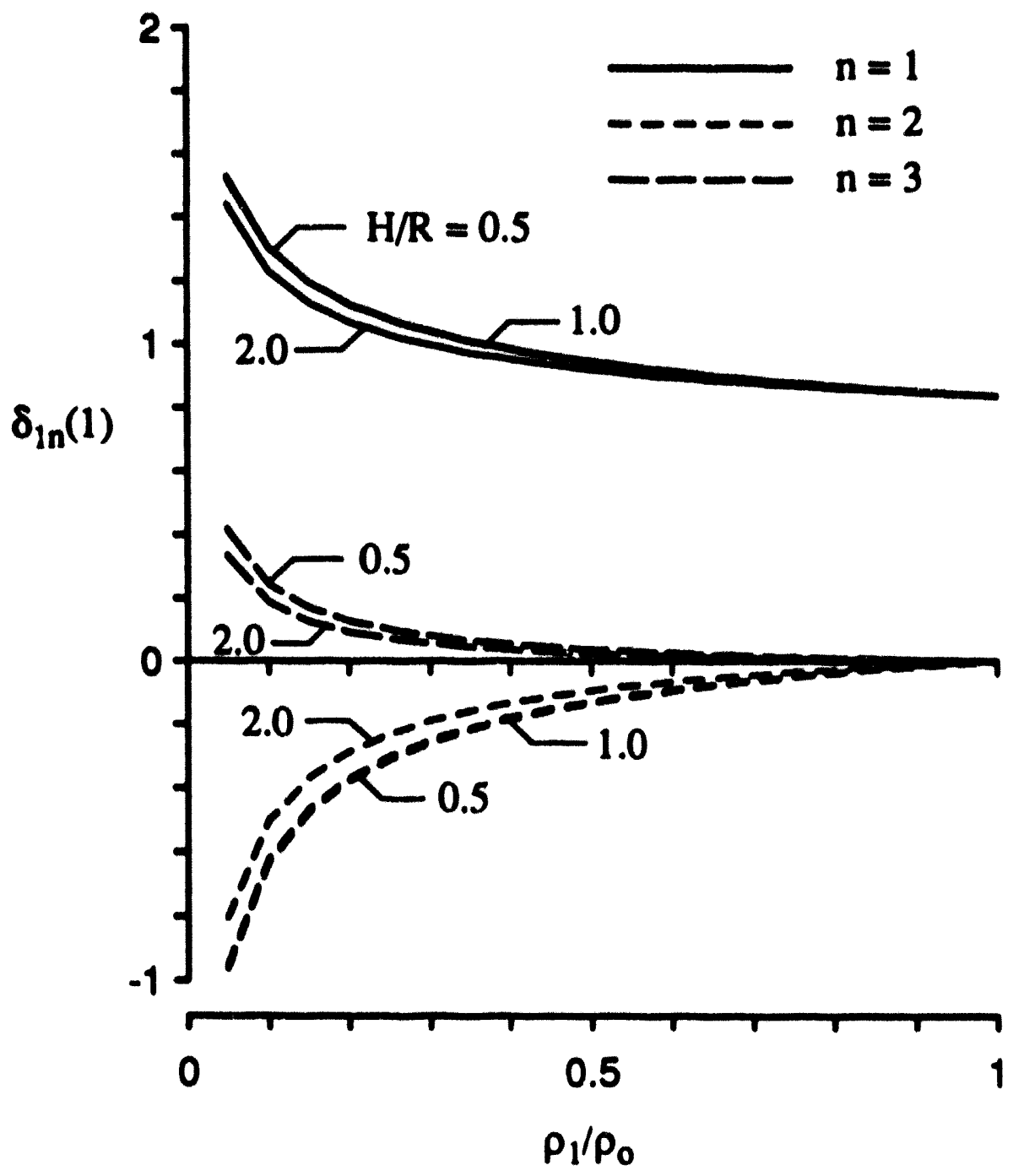

Figure 6.3 Surface displacement coefficients for fundamental horizontal mode of vibration of systems with different values of $H / R$ and $\rho_{1} / \rho_{0}$ 

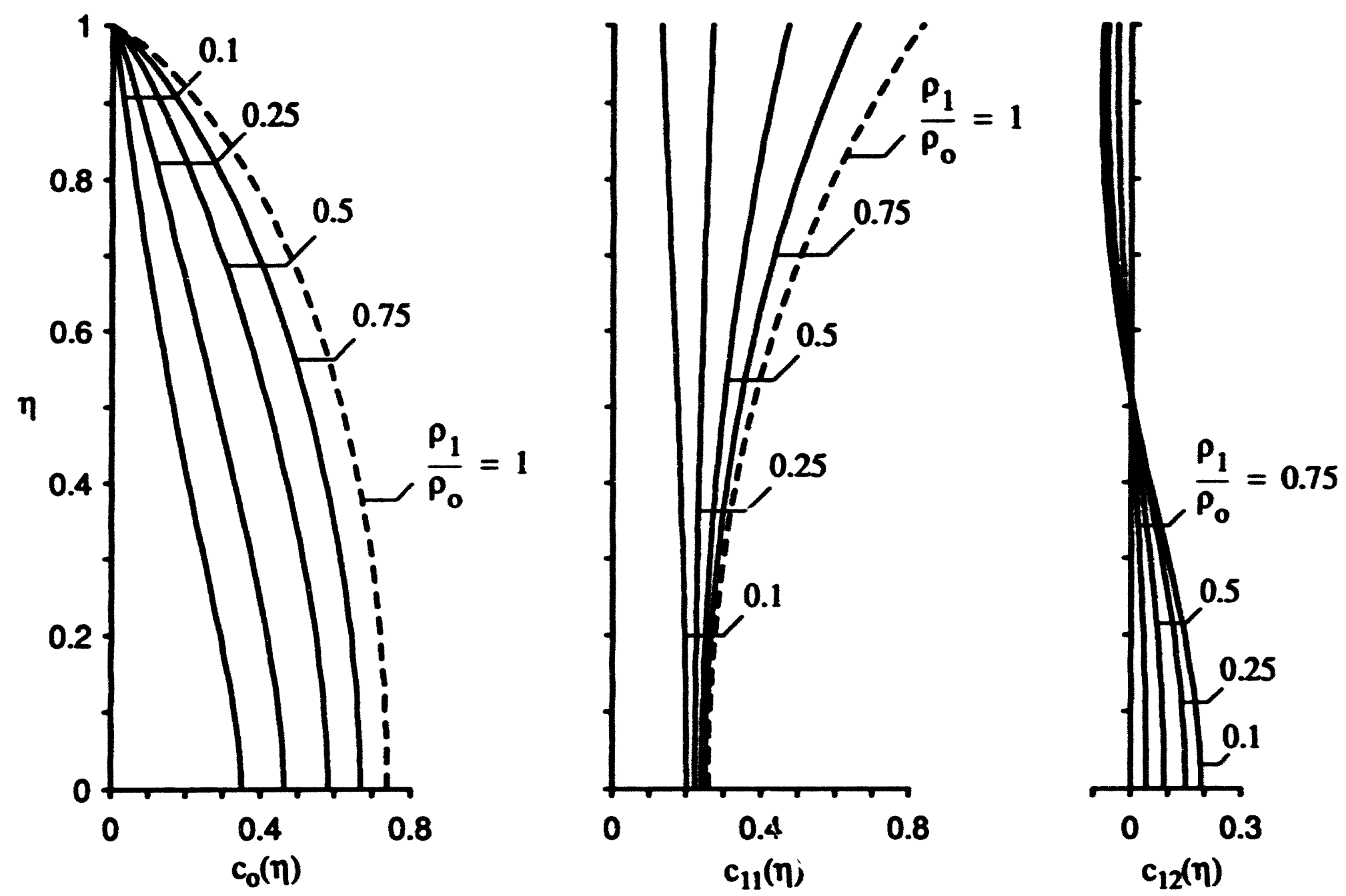

Figure 6.4 Effect of liquid density ratio $\rho_{1} / \rho_{0}$ on coefficients for impulsive and convective components of wall pressure for systems with $H / R=1.0$ 

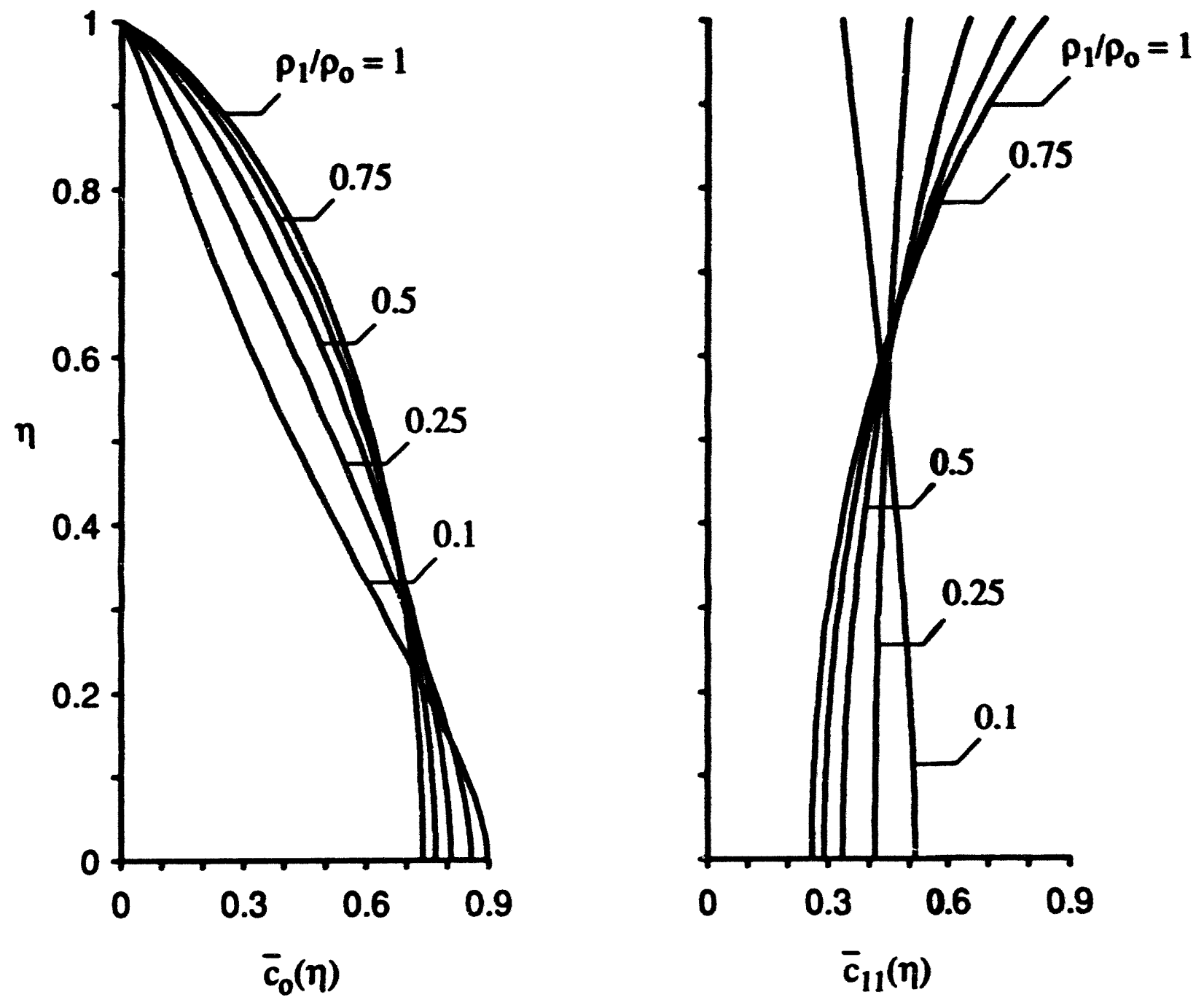

Figure 6.5 Coefficients $\bar{c}_{0}(\eta)$ and $\bar{c}_{11}(\eta)$ in expressions for impulsive and fundamental convective components of wall pressure for systems with $H / R=1.0$ 


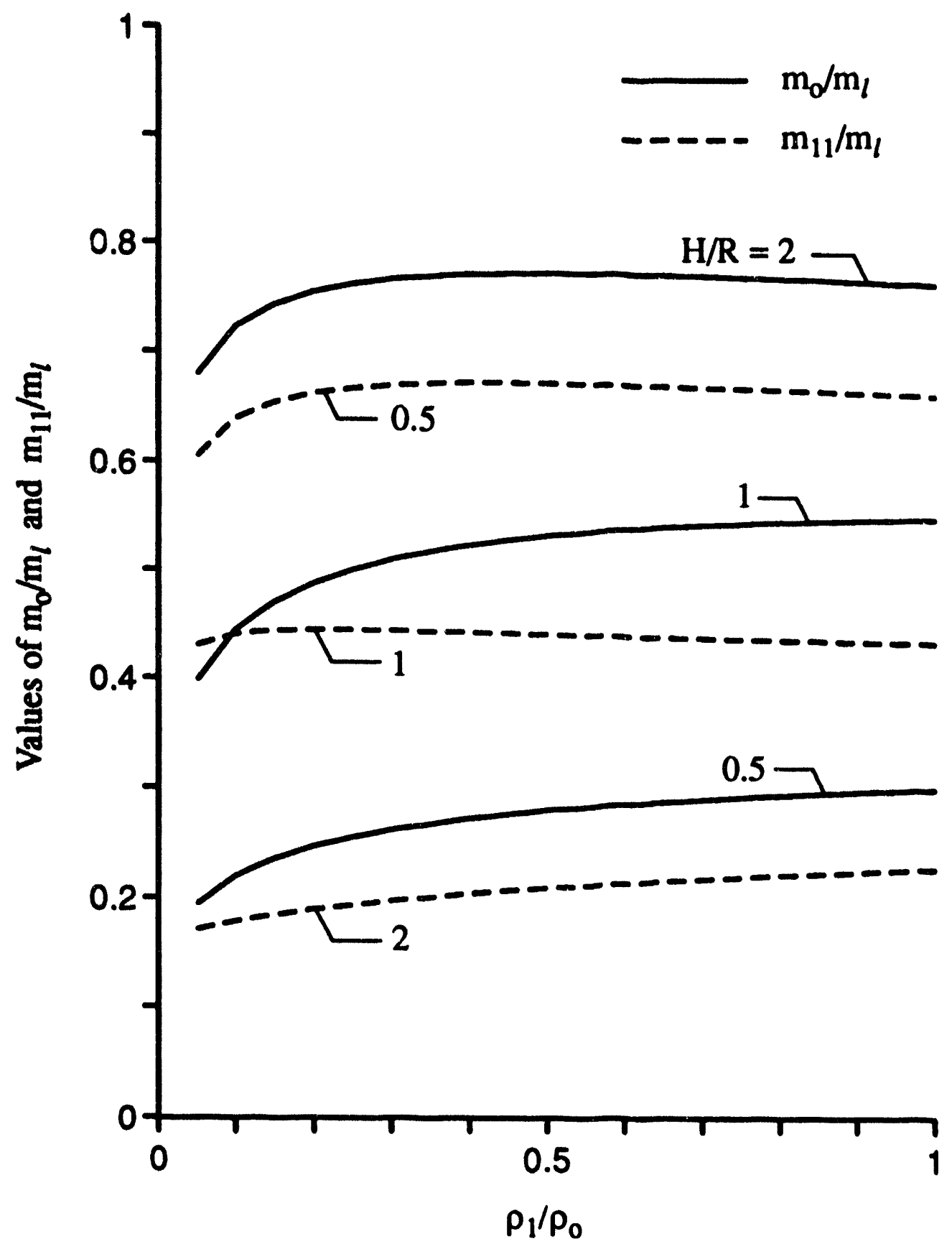

Figure 6.6 Normalized values of impulsive and fundamental convective masses for systems with different $H / R$ and $\rho_{1} / \rho_{0}$ 


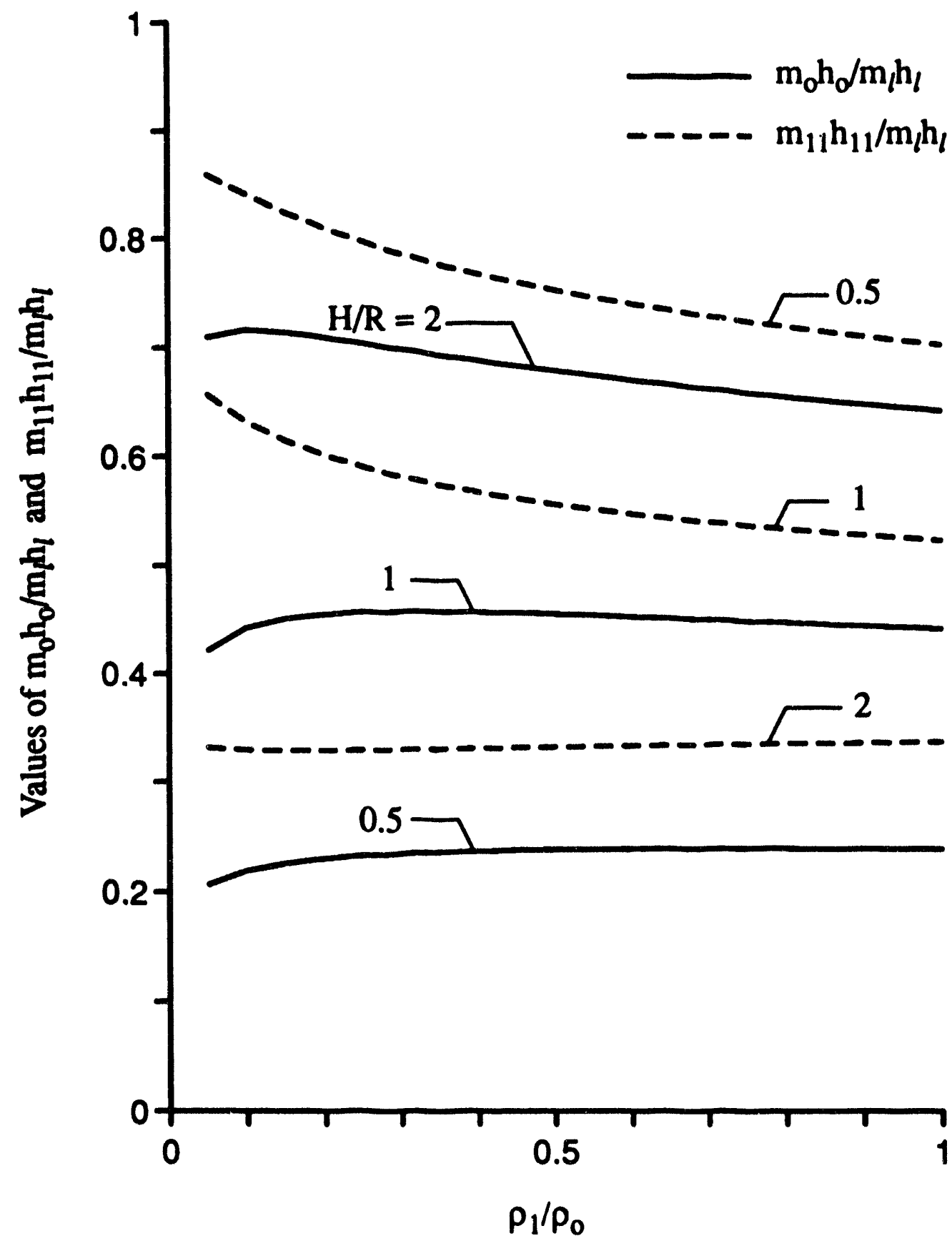

Figure 6.7 Normalized values of coefficients for impulsive and fundamental convective components of base moment for systems with different $H / R$ and $\rho_{1} / \rho_{0}$ 


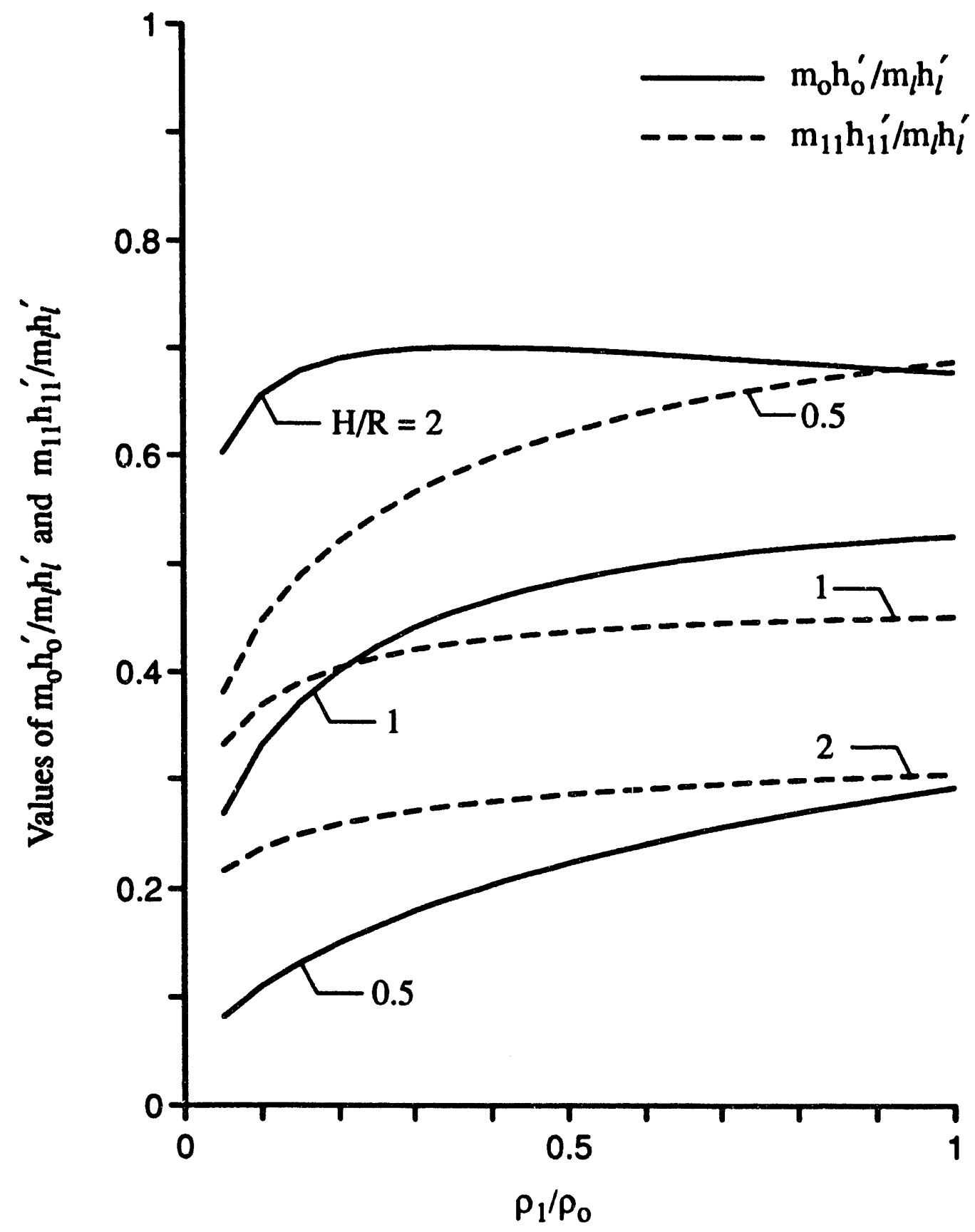

Figure 6.8 Normalized values of coefficients for impulsive and fundamental convective components of foundation moment for systems with different $H / R$ and $\rho_{1} / \rho_{0}$ 
Table 6.1: Values of $\gamma_{m n}$ for systems with different $H / R$ and $\rho_{1} / \rho_{o}$

\begin{tabular}{|c|c|c|c|c|c|c|}
\hline \multirow{3}{*}{$\rho_{1} / \rho_{o}$} & \multicolumn{6}{|c|}{ Values of $\gamma_{m n}$} \\
\hline & \multicolumn{3}{|c|}{$m=1$} & \multicolumn{3}{|c|}{$m=2$} \\
\hline & $n=1$ & $n=2$ & $n=3$ & $n=1$ & $n=2$ & $n=3$ \\
\hline & & & \multicolumn{2}{|c|}{$H / R=0.5$} & & \\
\hline 1 & 0.9206 & & & 2.6657 & & \\
\hline 0.75 & 0.7025 & 3.2241 & 6.3277 & 2.5200 & 3.1947 & 6.3218 \\
\hline 0.5 & 0.1668 & 3.3343 & 6.3894 & 2.3132 & 3.2691 & 6.3757 \\
\hline 0.25 & $0.8285^{*}$ & 3.5080 & 6.4918 & 1.9514 & 3.3953 & 6.4662 \\
\hline \multirow[t]{2}{*}{0.1} & $1.2237^{*}$ & 3.7127 & 6.6212 & 1.4393 & 3.5596 & 6.5825 \\
\hline & & & \multicolumn{2}{|c|}{$H / R=0.75$} & & \\
\hline 1 & 1.3809 & & & 3.9986 & & \\
\hline 0.75 & 1.2123 & 3.2171 & 6.3266 & 3.8547 & 3.1766 & 6.3157 \\
\hline 0.5 & 0.9475 & 3.3194 & 6.3868 & 3.6520 & 3.2265 & 6.3613 \\
\hline 0.25 & 0.1657 & 3.4837 & 6.4870 & 3.3038 & 3.3133 & 6.4386 \\
\hline \multirow[t]{2}{*}{0.1} & $0.9723^{*}$ & 3.6818 & 6.6141 & 2.8423 & 3.4320 & 6.5396 \\
\hline & & & \multicolumn{2}{|c|}{$H / R=1$} & & \\
\hline 1 & 1.8412 & & & 5.3314 & & \\
\hline 0.75 & 1.6880 & 3.2090 & 6.3251 & 5.1876 & 3.1653 & 6.3098 \\
\hline 0.5 & 1.4631 & 3.3017 & 6.3834 & 4.9849 & 3.1991 & 6.3472 \\
\hline 0.25 & 1.0314 & 3.4537 & 6.4806 & 4.6383 & 3.2582 & 6.4112 \\
\hline \multirow[t]{2}{*}{0.1} & $0.3663^{*}$ & 3.6422 & 6.6045 & 4.1802 & 3.3400 & 6.4958 \\
\hline & & & \multicolumn{2}{|c|}{$H / R=1.5$} & & \\
\hline 1 & 2.7618 & & & 7.9972 & & \\
\hline 0.75 & 2.6164 & 3.1931 & 6.3214 & 7.8533 & 3.1539 & 6.3007 \\
\hline 0.5 & 2.4105 & 3.2655 & 6.3747 & 7.6506 & 3.1713 & 6.3254 \\
\hline 0.25 & 2.0512 & 3.3886 & 6.4643 & 7.3040 & 3.2017 & 6.3678 \\
\hline \multirow[t]{2}{*}{0.1} & 1.5484 & 3.5497 & 6.5796 & 6.8459 & 3.2433 & 6.4246 \\
\hline & & & \multicolumn{2}{|c|}{$H / R=2$} & & \\
\hline 1 & 3.6824 & & & 10.6629 & & \\
\hline 0.75 & 3.5385 & 3.1803 & 6.3172 & 10.5190 & 3.1489 & 6.2950 \\
\hline 0.5 & 3.3358 & 3.2351 & 6.3648 & 10.3163 & 3.1593 & 6.3117 \\
\hline 0.25 & 2.9861 & 3.3303 & 6.4453 & 9.9697 & 3.1773 & 6.3404 \\
\hline 0.1 & 2.5208 & 3.4595 & 6.5502 & 9.5116 & 3.2016 & 6.3789 \\
\hline
\end{tabular}


Table 6.2: Surface displacement coefficients for systems with different $H / R$ and $\rho_{1} / \rho_{o}$

\begin{tabular}{|c|c|c|c|c|c|c|}
\hline \multirow{3}{*}{$\rho_{1} / \rho_{0}$} & \multicolumn{6}{|c|}{ Values of $\delta_{m n}(1)$} \\
\hline & \multicolumn{3}{|c|}{$m=1$} & \multicolumn{3}{|c|}{$m=2$} \\
\hline & $n=1$ & $n=2$ & $n=3$ & $n=1$ & $n=2$ & $n=3$ \\
\hline & & & \multicolumn{2}{|c|}{$H / R=0.5$} & & \\
\hline 1 & 0.8368 & & & 0.0729 & & \\
\hline 0.75 & 0.8790 & -0.0514 & 0.0132 & 0.0762 & -0.0038 & 0.0009 \\
\hline 0.5 & 0.9451 & -0.1345 & 0.0380 & 0.0814 & -0.0101 & 0.0026 \\
\hline 0.25 & 1.0787 & -0.3113 & 0.1014 & 0.0925 & -0.0242 & 0.0074 \\
\hline \multirow[t]{2}{*}{0.1} & 1.3039 & -0.6319 & 0.2440 & 0.1121 & -0.0509 & 0.0186 \\
\hline & & & \multicolumn{2}{|c|}{$H / R=0.75$} & & \\
\hline 1 & 0.8368 & & & 0.0729 & & \\
\hline 0.75 & 0.8788 & -0.0505 & 0.0126 & 0.0755 & -0.0028 & 0.0007 \\
\hline 0.5 & 0.9449 & -0.1327 & 0.0365 & 0.0797 & -0.0077 & 0.0020 \\
\hline 0.25 & 1.0791 & -0.3085 & $0.09 \$ 2$ & 0.0887 & -0.0189 & 0.0059 \\
\hline \multirow[t]{2}{*}{0.1} & 1.3065 & -0.6289 & 0.2383 & 0.1053 & -0.0413 & 0.0156 \\
\hline & & & \multicolumn{2}{|c|}{$H / R=1$} & & \\
\hline 1 & 0.8368 & & & 0.0729 & & \\
\hline 0.75 & 0.8778 & -0.0487 & 0.0119 & 0.0750 & -0.0021 & 0.0005 \\
\hline 0.5 & 0.9429 & -0.1286 & 0.0345 & 0.0782 & -0.0056 & 0.0015 \\
\hline 0.25 & 1.0766 & -0.3017 & 0.0940 & 0.0850 & -0.0138 & 0.0046 \\
\hline \multirow[t]{2}{*}{0.1} & 1.3055 & -0.6205 & 0.2306 & 0.0974 & -0.0307 & 0.0126 \\
\hline & & & \multicolumn{2}{|c|}{$H / R=1.5$} & & \\
\hline 1 & 0.8368 & & & 0.0729 & & \\
\hline 0.75 & 0.8737 & -0.0426 & 0.0100 & 0.0743 & -0.0011 & 0.0002 \\
\hline 0.5 & 0.9332 & -0.1140 & 0.0298 & 0.0764 & -0.0031 & 0.0008 \\
\hline 0.25 & 1.0589 & -0.2734 & 0.0835 & 0.0805 & -0.0076 & 0.0027 \\
\hline \multirow[t]{2}{*}{0.1} & 1.2825 & -0.5784 & 0.2107 & 0.0875 & -0.0167 & 0.0080 \\
\hline & & & \multicolumn{2}{|c|}{$H / R=2$} & & \\
\hline 1 & 0.8368 & & & 0.0729 & & \\
\hline 0.75 & 0.8684 & -0.0351 & 0.0081 & 0.0739 & -0.0007 & 0.0001 \\
\hline 0.5 & 0.9195 & -0.0949 & 0.0247 & 0.0755 & -0.0019 & 0.0004 \\
\hline 0.25 & 1.0290 & -0.2316 & 0.0718 & 0.0784 & -0.0046 & 0.0016 \\
\hline 0.1 & 1.2301 & -0.5035 & 0.1872 & 0.0830 & -0.0101 & 0.0052 \\
\hline
\end{tabular}


Table 6.3: Normalized values of effective masses in expression for base shear of systems with different $H / R$ and $\rho_{1} / \rho_{o}$

\begin{tabular}{|c|c|c|c|c|c|}
\hline$\frac{\rho_{1}}{\rho_{0}}$ & $\frac{\mathrm{m}_{\ell}}{\mathrm{m}_{\ell}}$ & $\frac{m_{11}}{m_{\ell}}$ & $\frac{m_{12}}{m_{l}}$ & $\frac{\mathrm{m}_{21}}{\mathrm{~m}_{\ell}}$ & $\frac{\mathrm{m}_{22}}{\mathrm{~m}_{\ell}}$ \\
\hline & & \multicolumn{2}{|c|}{$H / R=0.5$} & & \\
\hline 1 & 0.2999 & 0.6601 & & 0.0271 & \\
\hline 0.75 & 0.2926 & 0.6665 & 0.0015 & 0.0268 & 0.0001 \\
\hline 0.5 & 0.2804 & 0.6713 & 0.0085 & 0.0265 & 0.0008 \\
\hline 0.25 & 0.2559 & 0.6672 & 0.0320 & 0.0261 & 0.0030 \\
\hline \multirow[t]{2}{*}{0.1} & 0.2211 & 0.6396 & 0.0785 & 0.0256 & 0.0072 \\
\hline & & \multicolumn{2}{|c|}{$H / R=0.75$} & & \\
\hline 1 & 0.4391 & 0.5340 & & 0.0182 & \\
\hline 0.75 & 0.4320 & 0.5407 & 0.0016 & 0.0175 & 0.0001 \\
\hline 0.5 & 0.4181 & 0.5476 & 0.0089 & 0.0165 & 0.0007 \\
\hline 0.25 & 0.3866 & 0.5516 & 0.0331 & 0.0150 & 0.0027 \\
\hline \multirow[t]{2}{*}{0.1} & 0.3374 & 0.5405 & 0.0801 & 0.0135 & 0.0066 \\
\hline & & \multicolumn{2}{|c|}{$H / R=1$} & & \\
\hline 1 & 0.5475 & 0.4322 & & 0.0137 & \\
\hline 0.75 & 0.5435 & 0.4358 & 0.0016 & 0.0128 & 0.0001 \\
\hline 0.5 & 0.5323 & 0.4401 & 0.0092 & 0.0117 & 0.0006 \\
\hline 0.25 & 0.5009 & 0.4442 & 0.0341 & 0.0099 & 0.0021 \\
\hline \multirow[t]{2}{*}{0.1} & 0.4453 & 0.4408 & 0.0817 & 0.0080 & 0.0054 \\
\hline & & \multicolumn{2}{|c|}{$H / R=1.5$} & & \\
\hline 1 & 0.6858 & \multicolumn{2}{|l|}{0.3006} & 0.0091 & \\
\hline 0.75 & 0.6887 & 0.2972 & 0.0016 & 0.0083 & 0.0001 \\
\hline 0.5 & 0.6862 & 0.2927 & 0.0092 & 0.0072 & 0.0003 \\
\hline 0.25 & 0.6656 & 0.2866 & 0.0344 & 0.0056 & 0.0013 \\
\hline \multirow[t]{2}{*}{0.1} & 0.6145 & 0.2802 & 0.0826 & 0.0039 & 0.0033 \\
\hline & & \multicolumn{2}{|c|}{$H / R=2$} & & \\
\hline 1 & 0.7627 & 0.2270 & & 0.0068 & \\
\hline 0.75 & 0.7698 & 0.2193 & 0.0015 & 0.0062 & 0.0000 \\
\hline 0.5 & 0.7736 & 0.2092 & 0.0085 & 0.0052 & 0.0002 \\
\hline 0.25 & 0.7640 & 0.1940 & 0.0320 & 0.0039 & 0.0008 \\
\hline 0.1 & 0.7249 & 0.1789 & 0.0782 & 0.0025 & 0.0021 \\
\hline
\end{tabular}


Table 6.4: Normalized values of coefficients in expression for overturning moment at a section immediately above tank base of systems with different $H / R$ and $\rho_{1} / \rho_{0}$

\begin{tabular}{|c|c|c|c|c|c|}
\hline$\frac{\rho_{1}}{\rho_{0}}$ & $\frac{m_{0} h_{\ell}}{m_{\ell} h_{\ell}}$ & $\frac{m_{1} h_{\mu}}{m_{\ell} h_{\ell}}$ & $\frac{m_{12} h_{12}}{m_{\ell} h_{\ell}}$ & $\frac{\mathrm{m}_{21} \mathrm{~h}_{21}}{\mathrm{~m}_{\ell} \mathrm{h}_{\ell}}$ & $\frac{m_{22_{22}}}{m_{\ell} h_{\ell}}$ \\
\hline & & \multicolumn{2}{|c|}{$H / R=0.5$} & & \\
\hline 1 & 0.2394 & 0.7031 & & 0.0365 & \\
\hline 0.75 & 0.2398 & 0.7244 & -0.0190 & 0.0369 & -0.0014 \\
\hline 0.5 & 0.2388 & 0.7534 & -0.0431 & 0.0376 & -0.0030 \\
\hline 0.25 & 0.2332 & 0.7980 & -0.0753 & 0.0389 & -0.0050 \\
\hline \multirow[t]{2}{*}{0.1} & 0.2200 & 0.8417 & -0.0958 & 0.0410 & -0.0057 \\
\hline & & \multicolumn{2}{|c|}{$H / R=0.75$} & & \\
\hline 1 & 0.3520 & 0.6053 & & 0.0277 & \\
\hline 0.75 & 0.3550 & 0.6236 & -0.0185 & 0.0274 & -0.0010 \\
\hline 0.5 & 0.3566 & 0.6495 & -0.0418 & 0.0270 & -0.0022 \\
\hline 0.25 & 0.3524 & 0.6919 & -0.0722 & 0.0264 & -0.0034 \\
\hline \multirow[t]{2}{*}{0.1} & 0.3357 & 0.7384 & -0.0902 & 0.0258 & -0.0032 \\
\hline & & \multicolumn{2}{|c|}{$H / R=1$} & & \\
\hline 1 & 0.4425 & 0.5235 & & 0.0223 & \\
\hline 0.75 & 0.4495 & 0.5368 & -0.0177 & 0.0217 & -0.0007 \\
\hline 0.5 & 0.4559 & 0.5565 & -0.0397 & 0.0208 & -0.0015 \\
\hline 0.25 & 0.4574 & 0.5908 & -0.0677 & 0.0193 & -0.0022 \\
\hline \multirow[t]{2}{*}{0.1} & 0.4429 & 0.6326 & -0.0826 & 0.0175 & -0.0016 \\
\hline & & \multicolumn{2}{|c|}{$H / R=1.5$} & & \\
\hline 1 & 0.5664 & 0.4094 & & 0.0160 & \\
\hline 0.75 & 0.5800 & 0.4134 & -0.0152 & 0.0152 & -0.0004 \\
\hline 0.5 & 0.5954 & 0.4199 & -0.0337 & 0.0141 & -0.0008 \\
\hline 0.25 & 0.6111 & 0.4333 & -0.0556 & 0.0123 & -0.0011 \\
\hline \multirow[t]{2}{*}{0.1} & 0.6101 & 0.4540 & -0.0632 & 0.0100 & -0.0004 \\
\hline & & \multicolumn{2}{|c|}{$H / R=2$} & & \\
\hline 1 & 0.6445 & 0.3367 & & 0.0124 & \\
\hline 0.75 & 0.6609 & 0.3346 & -0.0124 & 0.0117 & -0.0002 \\
\hline 0.5 & 0.6811 & 0.3321 & -0.0270 & 0.0107 & -0.0005 \\
\hline 0.25 & 0.7061 & 0.3294 & -0.0428 & 0.0090 & -0.0006 \\
\hline 0.1 & 0.7181 & 0.3291 & -0.0432 & 0.0069 & -0.0001 \\
\hline
\end{tabular}


Table 6.5: Normalized values of coefficients in expression for foundation moment of systems with different $H / R$ and $\rho_{1} / \rho_{0}$

\begin{tabular}{|c|c|c|c|c|c|}
\hline$\frac{\rho_{1}}{\rho_{0}}$ & $\frac{m_{0} h_{l}^{\prime}}{m_{l} h_{l}^{\prime}}$ & $\frac{m_{11} h_{\mu \mu}^{\prime}}{m_{l} h_{l}}$ & $\frac{m_{12} h_{12}^{\prime}}{m_{\ell} h_{\ell}}$ & $\frac{m_{21} h_{21}^{\prime}}{m_{l} h_{l}^{\prime}}$ & $\frac{m_{22} h_{22}^{\prime}}{m_{l} h_{l}}$ \\
\hline & & \multicolumn{2}{|c|}{$H / R=0.5$} & & \\
\hline 1 & 0.2927 & 0.6869 & & 0.0131 & \\
\hline 0.75 & 0.2632 & 0.6616 & 0.0316 & 0.0118 & -0.0001 \\
\hline 0.5 & 0.2241 & 0.6212 & 0.0745 & 0.0101 & 0.0000 \\
\hline 0.25 & 0.1668 & 0.5454 & 0.1381 & 0.0079 & 0.0005 \\
\hline \multirow[t]{2}{*}{0.1} & 0.1113 & 0.4470 & 0.1967 & 0.0058 & 0.0013 \\
\hline & & \multicolumn{2}{|c|}{$H / R=0.75$} & & \\
\hline 1 & 0.4369 & 0.5402 & & 0.0148 & \\
\hline 0.75 & 0.4120 & 0.5319 & 0.0178 & 0.0134 & -0.0003 \\
\hline 0.5 & 0.3729 & 0.5150 & 0.0468 & 0.0115 & -0.0005 \\
\hline 0.25 & 0.3028 & 0.4748 & 0.0994 & 0.0087 & -0.0002 \\
\hline \multirow[t]{2}{*}{0.1} & 0.2195 & 0.4096 & 0.1595 & 0.0060 & 0.0006 \\
\hline & & \multicolumn{2}{|c|}{$H / R=1$} & & \\
\hline 1 & 0.5263 & 0.4508 & & 0.0149 & \\
\hline 0.75 & 0.5129 & 0.4465 & 0.0077 & 0.0135 & -0.0004 \\
\hline 0.5 & 0.4858 & 0.4373 & 0.0247 & 0.0117 & -0.0006 \\
\hline 0.25 & 0.4241 & 0.4133 & 0.0646 & 0.0089 & -0.0005 \\
\hline \multirow[t]{2}{*}{0.1} & 0.3324 & 0.3695 & 0.1218 & 0.0060 & 0.0003 \\
\hline & & \multicolumn{2}{|c|}{$H / R=1.5$} & & \\
\hline 1 & 0.6226 & 0.3575 & & 0.0131 & \\
\hline 0.75 & 0.6266 & 0.3519 & -0.0028 & 0.0120 & -0.0003 \\
\hline 0.5 & 0.6237 & 0.3428 & -0.0006 & 0.0105 & -0.0005 \\
\hline 0.25 & 0.5960 & 0.3246 & 0.0184 & 0.0081 & -0.0005 \\
\hline \multirow[t]{2}{*}{0.1} & 0.5246 & 0.2958 & 0.0629 & 0.0053 & 0.0001 \\
\hline & & \multicolumn{2}{|c|}{$H / R=2$} & & \\
\hline 1 & 0.6785 & 0.3048 & & 0.0110 & \\
\hline 0.75 & 0.6902 & 0.2972 & -0.0061 & 0.0101 & -0.0002 \\
\hline 0.5 & 0.7003 & 0.2858 & -0.0097 & 0.0089 & -0.0004 \\
\hline 0.25 & 0.6979 & 0.2652 & -0.0023 & 0.0069 & -0.0004 \\
\hline 0.1 & 0.6571 & 0.2369 & 0.0301 & 0.0046 & 0.0001 \\
\hline
\end{tabular}




\section{SECTION 7 CONCLUSIONS}

With the information presented herein, the free vibrational characteristics and the response to horizontal base shaking of rigid cylindrical tanks containing liquids of a density that decays exponentially with depth may be evaluated readily. The comprehensive numerical solutions that have been presented provide valuable insights into the underlying response mechanisms and into the effects and relative importance of the numerous parameters involved. The principal conclusions may be summarized as follows :

1. Unlike a homogeneous liquid, which for a given horizontal natural mode of vibration has a single vertical mode, the inhomogeneous liquid examined has an infinite number of such modes, each associated with a distinct frequency. The latter frequencies are smaller than the corresponding frequency of the homogeneous liquid.

2. For any horizontal mode of vibration, the $n$th vertical mode of the inhomogeneous liquid has $n-1$ zero crossings and its frequency decreases with increasing values of $n$.

3. For a specified horizontal mode of vibration, any two vertical modes satisfy the orthogonality relation defined by equation (43).

4. When normalized with respect to the pressures computed on the assumption that the entire liquid acts as a rigid mass, the coefficients in the expression for the impulsive and all convective components of the hydrodynamic wall pressures add up to unity. The same is also true of the corresponding coefficients for base shear and base moments in the tank.

5. The impulsive component of response may be obtained either by evaluating all the convective components and subtracting their sum from the response computed on the assumption that the entire liquid acts as a rigid mass, or, independently, without the prior evaluation of the convective effects.

6. When normalized with respect to the result computed on the assumption that the entire liquid in the tank acts as a rigid mass, the total hydrodynamic wall force or base shear for an inhomogeneous liquid with values of $\rho_{1} / \rho_{o}$ in the range 
between 1 and 0.25 may be considered to be equal to that for a homogeneous liquid. The same is also true of the moment induced above the tank base. These two effects may, therefore, be evaluated from well-established procedures for homogeneous liquids. This approximation, however, may not be adequate for the foundation moment, particularly for broad tanks with high gradients in liquid density.

7. The finite-difference representations of the equations for the response of the continuous system examined here are the same as the expressions for the response of the layered, discrete system studied in References 1 and 2. 


\section{SECTION 8}

\section{REFERENCES}

1. A. S. Veletsos and P. Shivakumar, 'Sloshing response of layered liquids in rigid tanks', Journal of Earthquake Engineering and Structural Dynamics Vol. 22, 801. 821 (1993); also published as BNL Report 52378, Brookhaven National Laboratory, Upton, New York, 1993

2. A. S. Veletsos, P. Shivakumar and K. Bandyopadhyay, 'Hydrodynamic effects in tanks containing layered liquids', BNL Report 52117, Brookhaven National Laboratory, Upton, New York, 1994

3. $Y$. Tang and $Y$. W. Chang, "The exact solutions to the dynamic response of tanks containing two liquids', Rep. ANL/RE-93/2, Argonne Nat. Lab., Argonne, III. 1993.

4. C. S. Yih, Stratified Flows, Academic Press Inc., New York, 1980, pp. 20-22.

5. Y. W. Chang, Discussion of 'Sloshing response of layered liquids in rigid tanks' by A. S. Veletsos and P. Shivakumar, to appear in Journal of Earthquake Engineering and Structural Dynamics.

6. A. S. Veletsos and P. Shivakumar, 'Reply to discussion by Y. W. Chang', to appear in Journal of Earthquake Engineering and Structural Dynamics.

7. H. Lamb, Hydrodynamics, Dover Publications, New York, 1945, pp. 378-380.

8. A. S. Veletsos, 'Seismic response and design of liquid storage tanks', Guidelines for the Seismic Design of Oil and Gas Pipeline Systems, Technical Council on Lifeline Earthquake Engineering, ASCE, New York, 1984, pp. 255-370 and 443-461. 


\section{SECTION $\theta$}

\section{NOTATION}

$a_{m 1, m 2}$ factors in expression for $e_{m}(\eta)$, defined by equation (68)

$A_{m}(t)$ instantaneous pseudoacceleration for $m$ th horizontal mode of vibration of homogeneous system

$A_{m n}(t)$ instantaneous pseudoacceleration for $m$ th horizontal and $n$th vertical mode of vibration of inhomogeneous system

$b_{1}, b_{2}$ factors in expression for $e_{m}(\eta)$, defined by equations (69) and (70) respectively

$c_{0}(\eta)$ dimensionless coefficient in expression for impulsive component of pressure, given by equation (72)

$\bar{c}_{0}(\eta)$ dimensionless coefficient in equation (88) for the impulsive component of wall pressure, defined by equation (90)

$c_{m n}(\eta)$ dimensionless coefficient in expression for convective component of pressure associated with $m$ th horizontal and $n$th vertical mode of vibration, given by equation (61)

$\bar{c}_{m n}(\eta)$ dimensionless coefficient in equation (88) for the convective component of wall pressure, defined by equation (90)

$C_{m} \quad$ dimensionless coefficient in expression for $\omega_{m}$

$C_{m n}$ dimensionless coefficient in expression for $\omega_{m n}$

$d$ vertical sloshing displacement of liquid at any point and time, defined by equation (5.3)

$D_{m}(\eta, t)$ vertical displacements of liquid along the tank wall for its $m$ th horizontal mode of vibration, given by equation (51)

$\hat{D}_{m n}(\eta)$ amplitudes of vertical displacements of liquid for its $m$ th horizontal and $n$th vertical natural mode of vibration

$e_{m}(\eta)$ dimensionless function in expressions for impulsive effects, defined by equation (62) and evaluated from equation (67)

$g$ acceleration due to gravity

$h_{l} \quad$ height of center of gravity of liquid mass from tank-base

$h_{0} \quad$ height of impulsive mass $m_{0}$ from tank-base

$h_{m n} \quad$ height of convective mass $m_{m n}$ from tank-base 


\begin{tabular}{|c|c|}
\hline$H$ & total depth of liquid in tank \\
\hline$J_{1}$ & Bessel function of first kind and first order \\
\hline$m_{1}$ & total liquid mass in tank \\
\hline$m_{0}$ & impulsive component of liquid mass, given by equation (77) \\
\hline$m n$ & $\begin{array}{l}\text { convective component of liquid mass associated with } m \text { th horizontal and } \\
n \text {th vertical mode of vibration, given by equation (78) }\end{array}$ \\
\hline$(t)$ & $\begin{array}{l}\text { instantaneous value of overturning moment at a section just above the } \\
\text { tank-base, given by equation ( } 80 \text { ) }\end{array}$ \\
\hline$M^{\prime}(t)$ & instantaneous value of foundation moment, given by equation (84) \\
\hline$p$ & hydrodynamic pressure, given by equation (57) \\
\hline$p^{i}$ & $\begin{array}{l}\text { impulsive component of hydrodynamic pressure, given by equation (59) or } \\
\text { equation (63) }\end{array}$ \\
\hline$p^{e}$ & convective component of hydrodynamic pressure, given by equation (60) \\
\hline$q_{m n}(t)$ & $\begin{array}{l}\text { time-dependent generalized coordinate corresponding to the } m \text { th horizon- } \\
\text { tal and } n \text {th vertical mode of vibration }\end{array}$ \\
\hline$Q_{b}(t)$ & instantaneous value of base shear, given by equation (76) \\
\hline$r$ & radial distance from tank-axis \\
\hline $\boldsymbol{R}$ & radius of cylindrical tank \\
\hline$t$ & time \\
\hline$\ddot{x}_{g}(t)$ & instantaneous value of frce-field ground acceleration \\
\hline 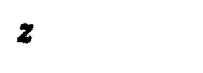 & vertical distance measured from tank-base \\
\hline$\beta$ & positive decay factor defining exponential variation in liquid density \\
\hline$\gamma_{m n}$ & dimensionless factor in expressions for $C_{m n}$ and $\hat{D}_{m n}(\eta)$ \\
\hline$\Gamma_{m n}$ & dimensionless factor defined by equation (48) \\
\hline $\begin{array}{l}\delta(\eta-1) \\
\delta_{m n}(\eta)\end{array}$ & $\begin{array}{l}\text { delta function, used to define density discontinuity at free-liquid surface } \\
\text { displacement coefficients in expression for } D_{m}(\eta, t) \text {, given by equation (52) }\end{array}$ \\
\hline$\epsilon_{m}$ & dimensionless factor defined by equation (69) \\
\hline$r$ & $=z / H=$ normalized vertical distance measured from tank-base \\
\hline$\theta$ & circumferential angle \\
\hline$\lambda_{m}$ & $m$ th root of $J_{1}^{\prime}(\lambda)=0$ \\
\hline $\boldsymbol{\xi}$ & $=r / R=$ dimensionless radial distance coordinate \\
\hline$\rho(z)$ & mass density of liquid, defined by equation (1) \\
\hline$\rho_{a v}$ & average value of mass density of liquid, defined by equation (89) \\
\hline$\rho_{1}$ & mass density of liquid at free liquid surface \\
\hline & mass density of liquid at tank-base \\
\hline
\end{tabular}


$\phi \quad$ velocity potential function, given by equation (10)

$\psi \quad$ velocity potential function associated with relative motion of liquid and tank wall, given by equation (19)

$\omega_{m} \quad$ circular natural frequency of homogeneous system for $m$ th horizontal sloshing mode of vibration

$\omega_{m n} \quad$ circular natural frequency of inhomogeneous system for $m$ th horizontal and $n$th vertical mode of vibration 

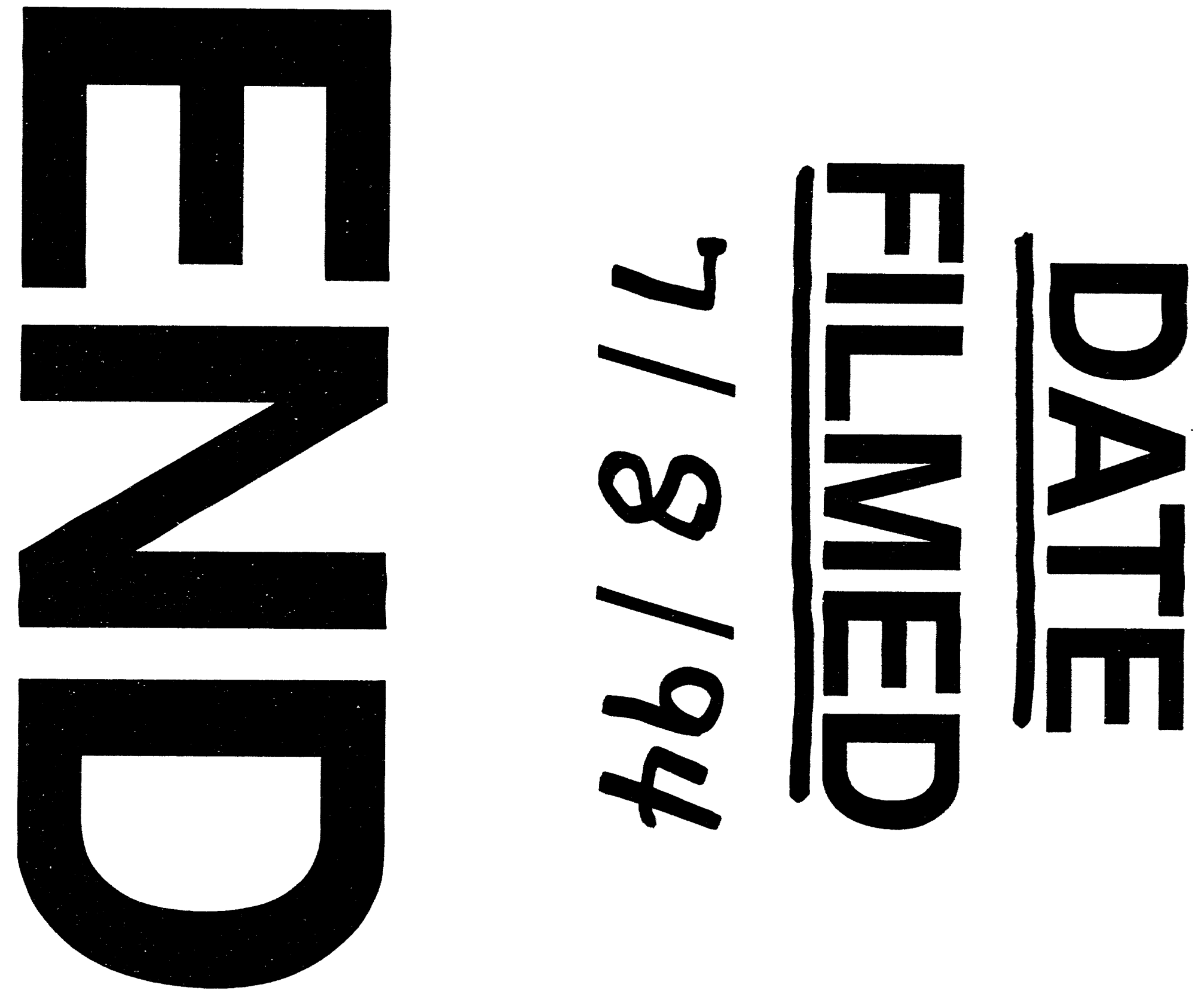
